\title{
I. PARITy ViOlation INDUCED BY WEAK NEUTRAL CURRENTS IN ATOMIC PHYSICS
}

\author{
M. A. BOUCHIAT \\ Laboratoire de Spectroscopie Hertzienne $\left({ }^{*}\right)$ \\ and \\ C. BOUCHIAT \\ Laboratoire de Physique Théorique (**), \\ Ecole Normale Supérieure, 24, rue Lhomond, 75231 Paris Cedex 05, France
}

(Reçu le 13 juin 1974)

\begin{abstract}
Résumé. - Nous présentons dans ce papier (le premier d'une série), une analyse générale des phénomènes de violation de la parité, induits par les courants neutres, en physique atomique. Nous donnons tout d'abord l'expression des potentiels électron-noyau et électron-électron à courte portée, violant la parité, prédits par les théories des interactions faibles contenant des courants neutres. Nous passons ensuite au calcul détaillé de l'élément de matrice du potentiel électron-noyau violant la parité entre des états $\mathrm{s}$ et $\mathrm{p}$ à une particule. Nous présentons une extension élaborée de la méthode utilisée par Foldy pour donner une base plus rigoureuse à la formule de Fermi-Segré donnant la valeur de la fonction d'onde d'un électron de valence dans un état s au niveau du noyau, et nous arrivons à une expression extrêmement simple, en fonction des énergies de liaison, avec une petite correction s'exprimant à l'aide du défaut quantique interpolé et du potentiel des électrons à l'origine. Nous indiquons les arguments qui nous permettent de penser que la précision de notre formule est comparable à celle de Fermi-Segré, c'est-à-dire quelques pour cents pour $Z \gg 1$. Un caractère remarquable de ce résultat, outre sa simplicité, est sa variation en $Z^{3}$ qui favorise de manière évidente les atomes lourds. Nous décrivons rapidement comment se manifeste le mélange de parité dans les transitions atomiques radiatives, en insistant particulièrement sur les conséquences de l'invariance par rapport au renversement du sens du temps qui est préservée par les interactions discutées dans ce papier. Pour améliorer les limites existantes, nous suggérons de chercher les effets de violation de la parité dans les transitions dipolaires magnétiques deux fois interdites, induites dans des atomes lourds par le faisceau d'un laser accordable. Dans un cas typique, tel que celui de la transition $6 \mathrm{~S}_{1 / 2} \rightarrow 7 \mathrm{~S}_{1 / 2}$ de la vapeur atomique de Césium, nous pouvons prédire un dichroïsme circulaire de l'ordre de $10^{-4}$. Nous terminons par une discussion sur les possibilités offertes, apparemment à plus long terme, par les atomes muoniques.
\end{abstract}

\begin{abstract}
In this paper (the first of a series), we present a general analysis of the parity violating phenomena induced by neutral currents in Atomic Physics. We first give expressions for the short range parity violating electron-nucleus and electron-electron potentials predicted by the theoretical schemes of weak interactions featuring neutral currents. We turn next to a detailed computation of the matrix element, between one-particle $s$ and $p$ states, of the electron-nucleus parity violating potential. Using a non-trivial extension of a method used by Foldy to put on a more rigorous basis a formula of Fermi and Segre giving the wave function of an $s$ valence electron at the nucleus, we arrive at a remarkably simple expression, in terms of the binding energies, with a small correcting term involving the interpolated quantum defect and the potential of the electronic cloud at the origin. Arguments are given which lead us to believe that the accuracy of our formula is comparable to that of the Fermi-Segre formula, i. e. a few per cent when $Z \gg 1$. Besides its simplicity, a remarkable feature of our result is a $Z^{3}$ dependence, which obviously favours heavy atoms. We give a brief analysis of the manifestation of parity mixing in atomic radiative transitions, with a particular
\end{abstract}

(*) Laboratoire associé au Centre National de la Recherche Scientifique.
(**) Laboratoire propre du C.N.R.S. associé à l'Ecole Normale Supérieure et à l'Université de Paris XI. 
emphasis on the implications of time reversal invariance, which is preserved by the interactions discussed in this paper. In order to improve the existing limits, we suggest looking for parity violating effects in twice forbidden magnetic dipole transitions induced by a tunable laser beam in heavy atoms. In a typical case, like the $6 \mathrm{~S}_{1 / 2} \rightarrow 7 \mathrm{~S}_{1 / 2}$ transition in atomic Cesium, a circular dichroism of the order of $10^{-4}$ is predicted. Finally we discuss the apparently more remote possibilities offered by the muonic atoms.

Introduction. - Recent developments in the field theory of weak interactions [1] have given a new dimension to the problem of the existence of weak neutral currents, i. e. of weak processes induced by the exchange of a weak neutral heavy boson. Until very recently all the known weak processes were adequately accounted for by a current-current lagrangian density of the type

$$
\mathcal{L}_{\mathrm{W}}(x)=\frac{G_{\mathrm{F}}}{\sqrt{2}} J_{\mu}^{+}(x) J^{\mu}(x),
$$

where $J_{\mu}(x)$ is a charged current, in the sense that it can be used as the source of a charged vector boson $\mathrm{W}^{ \pm}$. In fact $\mathcal{L}_{\mathrm{W}}(x)$ can be considered as a description of the exchange of a vector boson in the limit where its mass becomes infinite. Although there is not yet any experimental evidence for the existence of these heavy vector bosons (the present experimental limit on the mass of $\mathrm{W}^{ \pm}$is at least five nucleon masses), all the fashionable models of weak interactions assume the existence of vector bosons of finite mass as mediators of weak interactions. There are two reasons for this hypothesis : i) the current-current model leads to unphysical predictions in the limit of very high energy; ii) nobody knows how to compute higher order effects in a sensible way with a current-current lagrangian. The break-through which has occurred in the last few years consists in the fact that it is now possible to construct renormalizable field theory models, involving charged massive vector bosons. For the first time, it appears possible to build renormalizable field theory models unifying weak and electromagnetic interactions. Such a programme can be achieved only at the price of introducing new physical objects into the theory : neutral weak currents associated with the exchange of a neutral heavy vector boson $Z_{0}$, and/or new leptons, which, because of their assumed large masses, may have escaped observation.

The most popular model featuring neutral currents, the so-called Weinberg model [2], is a kind of a minimal model, in the sense that one avoids the introduction of new particles (at least in the lepton sector), and that it contains only one free angular parameter $\theta_{\mathrm{w}}$.

Processes induced by neutral weak currents in high energy neutrino-interactions have been under vigorous investigation during the last two years. In the heavy liquid bubble chamber Gargamelle exposed to the C.E.R.N. neutrino beam, a certain number of events have been found which have been interpreted as muonless neutrino-induced inelastic interactions in nuclei [3]. The most natural explanation of these events would be provided by the existence of a weak neutral current containing a neutrino and a hadronic component. Similar events have also been reported in an experiment performed at N.A.L. at much higher energies, where the background problems look very different [4]. Both results seem compatible with the existence of the neutral currents predicted by the Weinberg model, but other explanations involving some new feature of weak interactions (heavy leptons, etc...) cannot be absolutely excluded. We should also mention that the Gargamelle group has found three events which can be considered as possible candidates for elastic scattering of $\bar{v}_{\mu}$ on atomic electrons [5], a process which can be (unambiguously) attributed to the existence of neutral currents. If the statistics can be improved significantly, we shall be provided with clear evidence for weak neutral currents.

In this paper we shall present a detailed account of a theoretical investigation of those atomic physics effects associated with the existence of the electronic and hadronic components of the proposed weak neutral current. A brief account of this work has been given in a recent letter [6]. In the models of weak interactions discussed recently the coupling of the neutral heavy boson $Z_{0}$ to the electron and nucleons is parity violating, inducing a very short range parity violating interaction between the electron and the nucleus (a similar interaction between electrons is also present but, because of Coulomb effects, it will appear to be much harder to detect). The idea of looking for parity violating effects in atomic physics, as a way of investigating the possible existence of neutral weak currents is not a new one, but unfortunately up to now the theoretical investigations have been limited to the hydrogen atom where the effects expected are discouragingly small [7]. Stimulated both by the recent developments in weak interaction theory, and by the tunable laser revolution in experimental atomic physics, we have decided to take a fresh look at this problem. A little to our surprise, we have found that there is a reasonable hope of detecting the parity violating effects induced by weak neutral currents by exciting with a tunable laser beam twice forbidden magnetic dipole transitions $n \mathrm{~S}_{1 / 2} \rightarrow(n+1) \mathrm{S}_{1 / 2}$ in heavy atoms. Among other things, the feasibility of such an experiment is linked to the availability of a dye laser, tunable at the right frequency. 
The paper is divided into seven sections, followed by three appendices where we have gathered most of the technical details. We begin by a presentation of the parity violating electron-nucleon and electronelectron potentials, the latter playing a negligible role in the effect considered here (Section 1). We turn next to a computation of the matrix element of the parity violating potential between $s$ and $p$ states. This is performed using an extension of the Fermi-Segre formula. By means of a simple example, we show that the accuracy of the formula is not necessarily limited by the validity of a refined form of the J.W.K.B. approximation. We also give an expression for the rather large relativistic correction to be applied for $Z>10$. The detailed mathematical justification of the formulae presented in this second section is given in Appendices I and II. Next, we give a short review of parity mixing effects in radiative transitions, with an emphasis on the consequences of time reversal invariance (Section 3). In section 4, we make use of the results obtained previously to derive a simple formula which allows a quick order of magnitude estimate of parity violating effects in atoms. A review of the existing experimental results clearly indicates the need for more precise investigations (Section 5). In order to improve the situation, we propose studying twice forbidden magnetic dipole transitions, such as the S-S transitions in alkali atoms. The case of Cesium appears quite promising (parity effects of the order of $10^{-4}$ ). A complete analysis of the theoretical problems raised by the Cesium experiment will be given in a forthcoming publication. Some other experiments, involving Thallium and Lead, which could provide us with complementary information are also briefly discussed (Section 6). In the last section we explore the possibilities offered by the muonic atoms in the search for neutral weak currents. The situation appears much less favourable than one would have expected on the basis of a naive size effect argument, but will be improved by the gain in muon intensity to be expected with the arrival of the new generation of meson factories. Appendix III is devoted to a discussion of the electron-electron weak potential, which is shown to play a negligible role in the physical processes discussed in this paper.

1. The parity violating potential. - The parity violating electron-nucleus potential $V_{\text {p.v. }}^{\text {e.N. }}$ is associated with the exchange of a heavy neutral vector boson $Z_{0}$ between the electron and the nucleus, as illustrated in the diagram of figure 1.

We shall work in the limit of infinite boson mass and the momentum of the nucleus will be neglected. In the non-relativistic limit $V_{\text {p.v. }}^{\text {e.N. }}$ can be split into two parts, one independent of the nuclear spin, the other involving the nuclear spin. The ratio of the matrix elements of these two potentials is propor-

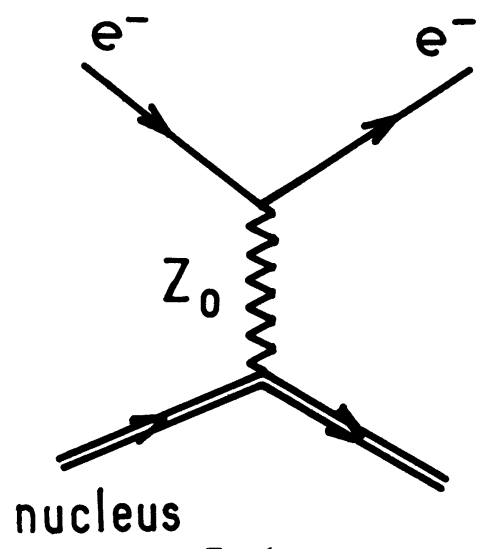

FIG. 1.

tional to a quantity having the following form :

$$
\frac{\left\langle\alpha \sum_{i=1}^{i=Z} \boldsymbol{\sigma}_{\mathrm{p}}^{i}+\beta \sum_{j=1}^{N} \boldsymbol{\sigma}_{\mathrm{n}}^{j}\right\rangle}{a Z+b N},
$$

where $\alpha, \beta, a, b$ are numbers of the order of unity, depending on the particular model of weak interactions, and $\sigma_{\mathrm{p}}, \sigma_{\mathrm{n}}$ are the spin operators of protons and neutrons. The numerator has a structure similar to the spin part of the nuclear magnetic moment which, because of the shell structure of the nuclei, does not increase with $Z$, but fluctuates between values associated with single particle states.

It is then clear that for the large values of $Z, Z>50$, which will concern us, the spin dependent part can be neglected in comparison with the spin independent part, except in special cases discussed briefly in the last section of this paper, where the effect to be observed is due entirely to the spin dependent part. Ignoring the nuclear spin effect, the parity violating potential reads :

$$
V_{\text {p.v. }}^{\text {e.N. }}=\frac{G_{\mathbf{F}}}{\sqrt{2}} \frac{Q_{\mathrm{W}}}{4 m_{\mathrm{e}} c}\left(\boldsymbol{\sigma} \cdot \mathbf{p} \delta^{3}(\mathbf{r})+\delta^{3}(\mathbf{r}) \boldsymbol{\sigma} \cdot \mathbf{p}\right),
$$

where $m_{\mathrm{e}}, \boldsymbol{\sigma}, \mathbf{p}, \mathbf{r}$ are respectively the mass, spin, momentum and position of the electron, $c$ is the velocity of light and $G_{\mathrm{F}}$ is the Fermi constant, which has the dimensions of an energy multiplied by a volume, and is conveniently given by :

$$
G_{\mathrm{F}} \simeq 3 \times 10^{-12} m_{\mathrm{e}} c^{2}\left(\frac{\hbar}{m_{\mathrm{e}} c}\right)^{3} .
$$

The dimensionless charge $Q_{\mathrm{w}}(Z, N)$ is model dependent. In fact, the above expression for $V_{\text {p.v. }}^{\text {e.N. is the }}$ most general form of a zero-range parity violating potential, with nuclear momentum and nuclear spin neglected, which is invariant under time reflexion.

The expression for the effective charge $Q_{\mathrm{w}}(Z, N)$ associated with the Weinberg model is derived in Appendix III, and found to be :

$$
Q_{\mathrm{w}}(Z, N)=-\left[\left(4 \sin ^{2} \theta_{\mathrm{w}}-1\right) Z+N\right] .
$$


If the muonless neutrino inelastic reactions observed at C.E.R.N. [3] and N.A.L. [4] are analyzed in the framework of this model, one finds, using reasonable dynamical assumptions $\left({ }^{1}\right)$, that $\sin ^{2} \theta_{\mathrm{w}}$ lies probably in the range 0.3 to 0.4 .

Even if the present evidence for a weak neutral current involving the neutrino is not confirmed, this would not necessarily imply that a neutral current involving the electron is not present. In fact, B. W. Lee, J. Prentki, and B. Zumino [8] have constructed a model of weak interactions where the neutral vector boson $Z_{0}$ is coupled only to charged leptons $\mathrm{e}^{-}, \mu^{-}$. The quantity $Q_{\mathrm{w}}(Z, N)$ is readily obtained and found to be :

$$
Q_{\mathrm{w}}(Z, N)=\sin ^{2} \theta_{\mathrm{w}} Z,
$$

where $\theta_{\mathrm{w}}$ is a parameter similar to that occurring in the Weinberg model, but on which we do not have any information.

One may also consider a situation where neutral weak currents are totally absent, but where their presence, with a considerably reduced strength, can be simulated by higher order effects. The quantity $Q_{\mathrm{w}}(Z, N)$ can be computed in renormalizable models without neutral currents [9]. The result depends strongly on the details of the models : masses of heavy leptons, etc... The only thing one can say is that $Q_{\mathrm{w}}(Z, N)$ is at least reduced by a factor of the order of $\alpha=1 / 137$ with respect to the values given by the previous models.

We would like now to say some words about the electron-electron short range parity violating potential. In reference [7] it is argued that because of the Coulomb repulsion, the two electrons are not affected by a zero-range potential. This argument is valid only if the two electrons have a small relative velocity, as can be seen most easily by looking at the absolute square of the ratio $\psi_{\text {coul }}(0) / \psi_{\text {plane }}(0)$, where $\psi_{\text {coul }}(\mathbf{r})$ is the Coulomb wave function of a system of the two electrons corresponding to the same relative velocity as the plane wave $\psi_{\text {plane }}(\mathbf{r})$ appearing in the denominator. Using a known expression for the Coulomb wave functions near the origin, one finds :

$$
\left[\frac{\psi_{\text {coul }}(0)}{\psi_{\text {plane }}(0)}\right]^{2}=\frac{2 \pi \alpha}{v / c} \frac{1}{\left(\exp \frac{2 \pi \alpha}{v / c}-1\right)}
$$

where $v / c$ is the relative velocity of the electrons. For light atoms $v / c$ is of the order of $\alpha$, and this ratio is of the order of a few per cent; in heavy atoms however, the Thomas-Fermi model tells us that $v / c$ is of the order of $\alpha Z^{2 / 3}$. If one takes, for instance, $Z=55$, the above ratio is found to be of the order

( $\left.{ }^{1}\right)$ The estimation given in the text is based on the parton model, which leads to a fair description of the high energy neutrino inelastic reactions induced by the charged currents. of unity, so that the Coulomb repulsion is no longer effective. In Appendix III, we have looked more carefully into this problem and have compared for heavy atoms the matrix elements of $V_{\text {p.v. }}^{\text {e.N. }}$ and $V_{\text {p.v. }}^{\text {e.e. }}$. For an atom with one valence electron, we have derived, in the framework of the Weinberg model, an effective one particle potential involving the electron density of the ion core $n_{\mathrm{e}}(\mathbf{r})$ :

$$
\begin{aligned}
& V_{\text {p.v. }}^{\text {e.e. }}=\frac{G_{\mathrm{F}}}{2 \sqrt{2} m_{\mathrm{e}} c}\left(1-4 \sin ^{2} \theta_{\mathrm{W}}\right) \times \\
& \times\left[\boldsymbol{\sigma} \cdot \mathbf{p} n_{\mathrm{e}}(\mathbf{r})+n_{\mathrm{e}}(\mathbf{r}) \boldsymbol{\sigma} \cdot \mathbf{p}\right] .
\end{aligned}
$$

In the case of Cesium ( $Z=55, N=78)$, we have found that the absolute value of the matrix element of the short range parity violating e-e potential is less than $2 \%$ of the corresponding quantity involving the electron-nucleus parity violating potential. This result can still be understood as a Coulomb effect, but this time it has to be attributed to an enhancement produced by the strong Coulomb attraction of the nucleus, of the electron wave function amplitude near the origin. In conclusion, we shall ignore in the rest of this paper the effect of the parity violating electron-electron interaction but, since it carries complementary information concerning the weak interaction models, we shall return to it in a future publication.

\section{Parity mixing in single particle wave functions. -} Under the influence of the parity violating potential parity mixing will occur between atomic wave functions. In the independent particle model of the atom, the electron-nucleus parity violating potential will mix, because of its zero-range nature, only $s$ and $p$ states.

We write the wave functions of $n s_{1 / 2}$ and $n^{\prime} \mathrm{p}_{1 / 2}$ as follows :

$$
\begin{gathered}
\Psi_{n \mathrm{~s}_{1 / 2}}(r)=R_{n 0}(r) \frac{1}{\sqrt{4 \pi}} \chi_{m}, \\
\psi_{n^{\prime} \mathrm{p}_{1 / 2}}(r)=R_{n^{\prime} 1}(r) \Omega_{1 / 2 m}^{1}(\theta, \varphi),
\end{gathered}
$$

where $\chi_{m}$ is the ordinary Pauli spinor and $\Omega_{j m}^{l}$ the spherical spinor :

$$
\Omega_{j m}^{l}=\sum_{m^{\prime}, \mu}\left\langle j m \mid l \mu \frac{1}{2} m^{\prime}\right\rangle Y_{l}^{\mu}(\theta, \varphi) \chi_{m^{\prime}}
$$

The matrix element of $V_{\text {p.v. }}$ is given by :

$$
\begin{aligned}
& \left\langle n \mathrm{~s}_{1 / 2}\left|V_{\text {p.v. }}^{\mathrm{e} . \mathrm{N} .}\right| n^{\prime} \mathrm{p}_{1 / 2}\right\rangle= \\
& \quad=\frac{3 i}{16 \pi m_{\mathrm{e}} c}\left(\frac{G_{\mathrm{F}}}{\sqrt{2}}\right) Q_{\mathrm{W}}(Z, N) R_{n^{\prime} 1}^{\prime}(0) R_{n 0}(0),
\end{aligned}
$$

where

$$
R_{n^{\prime} 1}^{\prime}(r)=\frac{\mathrm{d}}{\mathrm{d} r} R_{n^{\prime} 1}(r)
$$


Note that this matrix element is purely imaginary; as we shall see later this fact is connected with the time reversal invariance properties of $V_{\text {p.v. }}^{\text {e.N. }}$.

We shall now assume that $\Psi_{n s_{1 / 2}}(r)$ and $\Psi_{n^{\prime} \mathrm{p}_{1 / 2}}(r)$ are solutions of the Schrödinger equation with a central potential $V(r)$, satisfying the following properties :

a) when $r \rightarrow 0, r V(r) \rightarrow-e^{2} Z$ where $e Z$ is the electric charge on the atomic nucleus;

b) when $r>r_{\mathrm{c}}, V(r)=-\frac{\mathrm{z}_{0} e^{2}}{r}$.

In an alkali atom, $\mathrm{z}_{0}=1$ and $r_{\mathrm{c}}$ is interpreted as the radius of the ion core (the assumption of a sharp boundary to the electronic density of the core is convenient but by no means essential). Within these hypotheses a formula is available in the literature giving $R_{n 0}$ in terms of the interpolated quantum defects [10]. The derivation given by Fermi and Segré has been improved by Foldy [11] and, more recently, by Fröman and Fröman [12]. When used to compute hyperfine separations of atoms or ions with one s electron, the formula appears to be remarkably accurate, provided relativistic corrections are included properly. As an illustration we give a table obtained from results quoted in the book of H. G. Kuhn [10] :

$$
\begin{array}{cccccccc}
\text { Fermi-Segré } & \mathrm{Li} & \mathrm{Na} & \mathrm{K} & \mathrm{Ca}^{+} & \mathrm{Rb} & \mathrm{Cs} & \mathrm{Au} \\
\text { experiment } & 0.97 & 0.88 & 0.92 & -\overline{0} & - & - & - \\
& & & & & & &
\end{array}
$$

Recent measurements performed on several alkali excited states [13] also confirm the validity of the Fermi-Segré formula.

We have carried through an extension of the method of Foldy in order to get the starting coefficient of the radial wave function $\lim _{r \rightarrow 0} \frac{R_{n l}}{r^{l}}$. Although the final result remains rather simple, the derivation is fairly involved, and the details will be given in Appendix I. Here we shall only indicate the main points and illustrate them for a simple case.

For a potential satisfying conditions $a$ ) and $b$ ) and with some other minor technical assumptions, Ham [14] has shown that the solution of the Schrödinger equation, regular for $r=0$ and suitably normalized, $U_{l \varepsilon}(r)$, is an entire analytic function of the energy $\varepsilon$ ( $\varepsilon$ is the energy in atomic units). When $\varepsilon$ is not too far from zero, $U_{l \varepsilon}(r)$ is a slowly varying function of $\varepsilon$, so that a Taylor expansion around $\varepsilon=0$ can be limited to the first two terms, to a reasonable approximation. When $r>r_{\mathrm{c}}$, the quantum defect theory allows us to write $U_{l \varepsilon}(r)$ as a well-defined combination of regular and irregular Coulomb wave functions. In the particular case $l=\varepsilon=0, U_{l \varepsilon}(r)$ takes a rather simple form in terms of Bessel functions :

$U_{\varepsilon=l=0}=\sqrt{2 \rho}\left(J_{1}(\sqrt{8 \rho})-\operatorname{tg} \pi \mu(0) Y_{1}(\sqrt{8 \rho})\right)$, where $\rho=r / a_{0}$ is the radial coordinate in atomic units, and $\mu(\varepsilon)$ is the quantum defect, which, for $\varepsilon=-1 / v^{2}$ corresponding to a bound-state of energy $\varepsilon_{n}$, is given by

$$
\varepsilon_{n}=-\frac{1}{v_{n}^{2}}=-\frac{1}{\left(n-\mu\left(\varepsilon_{n}\right)\right)^{2}} .
$$

Seaton [15] has shown that the radial wave function $R_{n l}(r)$ is given in terms of $U_{l \varepsilon}(\rho)$ by :

$$
R_{n l}(r)=\frac{1}{a_{0}^{3 / 2}}\left(\Delta_{n l}\right)^{-1 / 2} \frac{1}{\rho} U_{\varepsilon_{n} l}(\rho),
$$

where $\Delta_{n l}$ is a non-analytic function of $\varepsilon$, given in terms of the quantum defect and its derivative, which, in the $l=0$ case and the limit of large $v_{n}$, takes the simple form :

$$
\begin{aligned}
& \Delta_{n, 0}=v_{n}^{3}\left(1+\left.\frac{\partial \mu}{\partial v}\right|_{v=v_{n}}\right) \times \\
& \times \frac{1}{\cos ^{2} \pi \mu\left(\varepsilon_{n}\right)}\left[1+O\left(\frac{1}{v_{n}^{3}}\right)\right] .
\end{aligned}
$$

Summarizing the above considerations, we may say that, within the hypotheses $a$ ) and $b$ ), a knowledge of the interpolated quantum defect $\mu(\varepsilon)$ provides us with an exact expression for the radial wave function for $r>r_{\mathrm{c}}$.

In the Foldy method, one starts with a representation of the wave function which is very accurate near the origin, but is normalized in an arbitrary way. The proper normalization is obtained by making the connection, for $r>r_{\mathrm{c}}$, with the known exact wave function given by the quantum defect. We shall sketch the method for the particular case $l=0$, $\varepsilon=0$. The general case is treated in Appendix I. The function $U(\rho)=U_{00}(\rho)$ is the regular solution of the simple differential equation

$$
\left(\frac{\mathrm{d}^{2}}{\mathrm{~d} \rho_{2}}+v(\rho)\right) U(\rho)=0,
$$

where $v(\rho)=\frac{V(r)}{-e^{2} / 2 a_{0}}$ is such that $\rho v(\rho) \rightarrow 2 Z$ when $\rho \rightarrow 0$ and $\rho v(\rho) \rightarrow 2$ when $\rho \rightarrow \infty$.

The basic idea of the method is to consider a representation of $U(\rho)$ of the following form :

$$
U(\rho)=C\left(\varphi^{\prime}(\rho)\right)^{-1 / 2} \sqrt{8 \varphi} J_{1}(\sqrt{8 \varphi}),
$$

where the function $\varphi$ obeys the non-linear differential equation :

$$
\frac{2 \varphi^{\prime 2}}{\varphi}=v(\rho)+D \varphi,
$$

with

$$
\operatorname{D} \varphi=\frac{3}{4}\left(\frac{\varphi^{\prime \prime}}{\varphi^{\prime}}\right)^{2}-\frac{1}{2}\left(\frac{\varphi^{\prime \prime \prime}}{\varphi^{\prime}}\right),
$$

and $C$ is a constant to be determined. 
The choice of the above representation is motivated by the fact that, in the case $v(\rho)=2 Z / \rho$, the regular solution is obtained by taking $\varphi \equiv Z \rho$, which implies $\mathfrak{D} \varphi \equiv 0$. One expects, then, that the use of the approximate solution (13) obtained by ignoring in (14) the term $\mathfrak{D} \varphi$, is justified near the origin. For large values of $\rho$ the approximation $2 \varphi^{\prime 2} / \varphi \approx v(\rho)$ coincides with the ordinary J. W. K. B. result, as can be easily verified if one uses the asymptotic form of the Bessel functions for large argument. Since the J. W. K. B. approximation is known to give correct results for Coulomb functions when the two conditions $\rho \gg 1$ and $\rho \ll 1 / \varepsilon$ are satisfied simultaneously, the approximation $\mathfrak{D} \varphi \approx 0$ will also be valid. In fact one can verify, using the asymptotic form of the Bessel functions, that the expression (8) can be cast in the form (13), for large values of $\rho$, if one takes :

$$
\sqrt{8 \varphi(\rho)}=\sqrt{8 \rho}+\pi \mu(0)+O(1 / \rho) .
$$

The ratio $\operatorname{D} \varphi / v(\rho)$ is then found to be :

$$
\frac{D \varphi}{v(\rho)}=\frac{D \varphi}{2 / \rho}=-\frac{3}{64} \frac{\pi \mu(0)}{\rho \sqrt{\rho}}+O\left(\frac{1}{\rho^{2}}\right) .
$$

The approximation $\mathrm{D} \varphi \approx 0$ appears as a refined J. W. K. B. approximation, having the great advantage of being valid near the potential pole at the origin. Moreover, in the case $l>0$, the usual difficulty near the classical turning point is completely avoided.

In the intermediate region $\rho \sim 1$, the approximation $\mathfrak{D} \varphi \approx 0$ is probably no longer very accurate, but in fact it is not needed in order to obtain the Fermi-Segré fromula. What is needed is a weaker assumption :

The wave function $U(\rho)$ can be represented by the expression (13) involving a function $\varphi$ which remains regular when $\rho \sim 1$ and which admits an asymptotic expansion which coincides with the formal series

$$
\varphi=\varphi_{0}+\varphi_{1}+\cdots+\varphi_{n},
$$

constructed through an iterative procedure starting with $\mathfrak{D} \varphi=0$. We give below the first two terms of the iteration :

$$
\begin{aligned}
\sqrt{8 \varphi_{0}} & =\int_{0}^{\rho} \sqrt{v(\rho)} \mathrm{d} \rho \\
\varphi_{1} & =\sqrt{\frac{\varphi_{0}}{8}} \int_{0}^{\rho} \frac{D \varphi_{0}}{\sqrt{v(\rho)}} \mathrm{d} \rho .
\end{aligned}
$$

In order to perform the connection between the two forms, (8) and (13), of the wave function, let us look again in the region $\rho>\rho_{\mathrm{c}}$, with $\rho \gg 1$. By integrating the two sides of equation (14), one obtains :

$$
\sqrt{8 \varphi}=\int_{0}^{\rho} \sqrt{8 \varphi+D \varphi} d \varphi .
$$

The behaviour of $\mathfrak{D} \varphi$ for large values of $\rho$, exhibited in eq. (17), implies the convergence of the integral

$$
\int_{0}^{\infty}(\sqrt{v(\rho)+\mathscr{D} \varphi}-\sqrt{v(\rho)}) \mathrm{d} \rho .
$$

It is then possible to rewrite (19) as :

$$
\begin{aligned}
& \sqrt{8 \varphi}=\int_{0}^{\infty}[\sqrt{v(\rho)+\operatorname{D} \varphi}-\sqrt{v(\rho)}] \mathrm{d} \rho+ \\
& +\int_{\infty}^{\rho}(\sqrt{v(\rho)+\mathfrak{D} \varphi}-\sqrt{v(\rho)}) \mathrm{d} \rho \\
& +\int_{0}^{\rho} \sqrt{v(\rho)} \mathrm{d} \rho
\end{aligned}
$$

Remembering that for $\rho>\rho_{\mathrm{c}}, v(\rho)=2 / \rho$, we obtain, after some simple manipulation, the following expression for $\varphi$, valid when $\rho>\rho_{\mathrm{c}}$ :

$$
\begin{aligned}
\sqrt{8 \varphi}=\Lambda+\delta \Lambda & +\sqrt{8 \rho}+ \\
& +\int_{+\infty}^{\rho}\left(\sqrt{\frac{2}{\rho}+\mathfrak{D} \varphi}-\sqrt{\frac{2}{\rho}}\right) \mathrm{d} \rho,
\end{aligned}
$$

with the following values for the constants $\Lambda$ and $\delta \Lambda$ :

$$
\left.\begin{array}{c}
\Lambda=\int_{0}^{\rho_{\mathrm{c}}}\left(\sqrt{v(\rho)}-\sqrt{\frac{2}{\rho}}\right) \mathrm{d} \rho \\
\delta \Lambda=\int_{0}^{\infty}(\sqrt{v(\rho)+D \varphi}-\sqrt{v(\rho)}) \mathrm{d} \rho
\end{array}\right\} .
$$

When $\rho \gg 1$, the correcting term,

$$
\int_{\infty}^{\rho}\left(\sqrt{\frac{2}{\rho}+\mathcal{D} \varphi}-\sqrt{\frac{2}{\rho}}\right) d \rho,
$$

goes to zero like $1 / \rho$, so we have for large values of $\rho$ :

$$
\sqrt{8 \varphi}=\Lambda+\delta \Lambda+\sqrt{8 \rho}+O\left(\frac{1}{\rho}\right) \text {. }
$$

Again in the limit of large $\rho$, the two expressions (8) and (13) for the function $U(\rho)$ can be written in a similar form, if one makes use of the asymptotic formulae for the Bessel functions :

$$
\begin{aligned}
(8) \Rightarrow & U(\rho)=\frac{1}{2 \cos \pi \mu(0)}\left(\frac{z}{2 \pi}\right)^{1 / 2} \times \\
& \times\left[\cos \left(z+\pi \mu(0)-\frac{3 \pi}{4}\right)+O\left(\frac{1}{z}\right)\right], \\
(13) \Rightarrow & U(\rho)=C\left(\frac{z}{2 \pi}\right)^{1 / 2} \times \\
\times & {\left[\cos \left(z+\Lambda+\delta \Lambda-\frac{3 \pi}{4}\right)+O\left(\frac{1}{z}\right)\right], }
\end{aligned}
$$


where we have introduced the variable $z=\sqrt{8 \rho}$. The above expressions coincide provided one makes the identification :

$$
C=(-1)^{\kappa} \frac{1}{2 \cos \pi \mu(0)}, \quad \pi \mu(0)=\Lambda+\delta \Lambda+k \pi,
$$

where $k$ is an arbitrary integer. If one takes the limiting case of atomic hydrogen, which means that $\frac{v(\rho)}{2 / \rho} \rightarrow 1$ uniformly in $\rho$, the constants $\Lambda$ and $\delta \Lambda$ go to zero. But, by definition, $\mu=0$ for atomic hydrogen. The choice $k=0$ appears then the only possible one. Thus we have finally :

$$
\left.\begin{array}{rl}
C & =\frac{1}{2 \cos \pi \mu(0)} \\
\pi \mu(0) & =\Lambda+\delta \Lambda
\end{array}\right\} .
$$

Remembering that $\varphi(\rho) / Z \rho \rightarrow 1$ when $\rho \rightarrow 0$, one obtains immediately, using (10), (11), (13) and (23), the radial $\mathrm{s}$ wave function at the origin :

$$
R_{n 0}(0)=\frac{2 Z^{1 / 2}}{a_{0}^{3 / 2}}\left[v_{n}^{3}\left(1+\frac{\partial \mu}{\partial v}\right)\right]^{-1 / 2}(1+O(\varepsilon)) .
$$

In order to arrive at this formula, it is not at all necessary to assume that $\mathfrak{D} \varphi$ is small in the intermediate region $\rho \sim 1$. The corrections arising from the finite value of $\mathscr{D} \varphi$ appear only in the quantity $\delta \Lambda$ and will be automatically included in the Fermi-Segré formula provided one uses the empirical quantum defect.

With similar assumptions, the method sketched out above has been extended in two ways : first, it can be properly generalized to values $l>0$ in order to give the derivative of the $\mathrm{p}$ wave radial function at the origin; secondly, the first order correction in the reduced energy $\varepsilon$ can be included in the results, but in order to do this in a consistent way, it appears necessary to take explicitly into account the first order correction in $\mathfrak{D} \varphi$ in the region of large $\rho$. Although the basic ideas remain the same as in the simple case treated above, the algebra is much more involved. All the details can be found in Appendix I, and only the final result will be quoted here :

$$
\begin{aligned}
R_{n 0}(0) & =\frac{2 Z^{1 / 2}}{a_{0}^{3 / 2} v_{n}^{3 / 2}}\left(1+\delta_{0}\left(\varepsilon_{n}\right)\right), \\
\left.\frac{\partial R_{n 1}}{\partial r}\right|_{r=0} & =\frac{2}{3} \frac{Z^{3 / 2}}{a_{0}^{5 / 2} v_{n}^{3 / 2}}\left(1+\delta_{1}\left(\varepsilon_{n}\right)\right) .
\end{aligned}
$$

The quantities $\delta_{0}\left(\varepsilon_{n}\right)$ and $\delta_{1}\left(\varepsilon_{n}\right)$, which are smaller than unity when $\varepsilon \ll 1$ and $Z \gg 1$, are given by :

$$
\begin{aligned}
1+\delta_{0}(\varepsilon)= & \left(1+\frac{\partial \mu(\varepsilon)}{\partial v}\right)^{-1 / 2}\left(1+O\left(\varepsilon^{2}\right)\right) \\
1+\delta_{1}(\varepsilon)= & \left(1+\frac{\partial \mu(\varepsilon)}{\partial v}\right)^{-1 / 2} \times \\
& \quad \times\left[1-\zeta+O\left(\varepsilon^{2}\right)+O\left(\zeta^{2}\right)\right]
\end{aligned}
$$

where $\mu(\varepsilon)$ is the interpolated quantum defect. The quantity $\zeta$, which appears only for $p$ waves, involves the potential of the electronic cloud at the nucleus, $V_{\mathrm{e}}(0)$ :

$$
\zeta=\frac{V_{\mathrm{e}}(0)}{m_{\mathrm{e}} c^{2} \alpha^{2}} \frac{1}{Z^{2}}-\frac{\varepsilon}{2 Z^{2}} .
$$

For heavy atoms an approximate expression for $\zeta$ can be obtained with the help of the Thomas-Fermi model :

$$
\zeta \approx \frac{1.8}{Z^{2 / 3}}-\frac{\varepsilon}{2 Z^{2}}
$$

so that expansion in terms of $\zeta$ is certainly adequate when $Z \gg 1$.

We would like to emphasize once more that the accuracy of the above formulae is not limited by the fact that we have used our modified J. W. K. B. approximation in a region where its validity is rather uncertain. The essential point in the derivation is that the function $\varphi(\rho)$ can be continued from the region $\rho \approx 0$ to the region $\rho>\rho_{\mathrm{c}}$. The main role of the modified J. W. K. B. approximation is to provide us with an argument for the possibility of such a continuation.

In order to test the accuracy of our formula, we have compared the results obtained by using it with the atomic p-wave functions obtained from HartreeFock calculations [16] [17]. Our formula for p-wave functions is supposed to work only for valence p-electrons in atoms of high $Z$, when the binding energy $\varepsilon_{n}$, in atomic units, is $\ll 1$. We have made the comparison in the cases of Gallium, Indium and Thallium. In the application of the formula one should use the calculated binding energies rather than the experimental ones. The main source of uncertainty is the factor involving the derivative of the quantum defect. We have found that our formula works with an accuracy of the order of $10 \%$. In conclusion, we expect that, within the framework of the independent particle description of the atoms (which is certainly a fairly good approximation for alkali atoms), our formulae are correct to within $10 \%$ when applied to states of the valence electron.

We are now in a position to write down the matrix element of the parity violating potential :

$$
\begin{aligned}
& \left\langle n \mathrm{~s}_{1 / 2}\left|V_{\text {p.v. }}\right| n^{\prime} \mathrm{p}_{1 / 2}\right\rangle= \\
& =i \frac{\hbar G_{\mathrm{F}} / \sqrt{2} Z^{2} Q_{\mathrm{W}}(Z, N)}{4 \pi m_{\mathrm{e}} c a_{0}^{4}\left(v_{n} v_{n^{\prime}}\right)^{3 / 2}}\left(1+\delta_{0}\left(\varepsilon_{n}\right)\right)\left(1+\delta_{1}\left(\varepsilon_{n^{\prime}}\right)\right),
\end{aligned}
$$

where $\varepsilon_{n}=-1 / v_{n}^{2}$ and $\varepsilon_{n^{\prime}}=-1 / v_{n^{\prime}}^{2}$ are the binding energies in Rydberg units. 
The formula (29) still cannot be used in this form, since it does not contain any relativistic corrections. These corrections are expected to be quite substantial for atoms of large $Z$, especially if we remember that Dirac wave functions are infinite at the origin for $\mathrm{s}_{1 / 2}$ and $\mathrm{p}_{1 / 2}$ electrons moving in the Coulomb potential of a point charge. In Appendix II, we give a derivation of the relativistic correction factor $K_{\mathrm{r}}$ by which we should multiply the formula (29). We follow a prescription which has been quite successful in $\beta$-decay calculations and which consists in using Dirac wave functions for a point like nucleus, but evaluated at the nuclear radius.

The correction factor $K_{\mathrm{r}}$ is given as a function of the nuclear radius $R$ :

$$
\begin{gathered}
K_{\mathrm{r}}(Z, R) \approx\left[\frac{\Gamma(3)}{\Gamma\left(2 \sqrt{1-(Z \alpha)^{2}}+1\right)} \times\right. \\
\left.\times\left(\frac{2 Z R}{a_{0}}\right)^{\left(\sqrt{1-Z \alpha)^{2}}-1\right)}\right]^{2} .
\end{gathered}
$$

$R$ can be evaluated from the following empirical formula (18), valid for $Z>40$ :

$$
R \simeq 1.2 A^{1 / 3} \times 10^{-13} \mathrm{~cm} .
$$

To illustrate the importance of $K_{\mathrm{r}}$, we quote the values of $K_{\mathrm{r}}$ for Cesium ${ }^{133}$ and Lead $^{208}$ :

$$
K_{\mathrm{r}}(\mathrm{Cs}) \simeq 2.8, \quad K_{\mathrm{r}}(\mathrm{Pb}) \simeq 9.0 .
$$

To conclude this section, we would like to emphasize a feature of our results which is of great practical importance, namely the fact that the matrix element of the parity violating potential increases roughly like $Z^{3}$, which obviously favours heavy atoms in the search for parity violating effects.

\section{Effects on radiative transitions of parity mixing} in atomic wave functions. - The mixing of wave functions of different parity will manifest itself by typical parity violating effects in physical processes involving absorption and emission of radiation by atoms.

Similar effects have been studied extensively in nuclear physics and recently unambiguous proof of the existence of a parity violating component in nuclear forces has been found [19]. Although the basic formula given below is in the literature, we have, for the sake of completeness, included a simplified derivation of it, with emphasis on the consequences of the time invariant properties of the parity violating potential discussed in this paper.

The interaction of an atom with the radiation field is given in the dipole approximation by :

$$
H_{1}^{\mathrm{em}}=- \text { d.E }(0)-\boldsymbol{\mu} . \mathbf{B}(0) .
$$

In this expression, the quantities :

$$
\left.\begin{array}{l}
\mathbf{d}=\int \rho(\mathbf{r}) \mathbf{r} \mathrm{d}^{3} r, \\
\boldsymbol{\mu}=\frac{1}{2} \int \mathbf{r} \wedge \mathbf{j}(\mathbf{r}) \mathrm{d}^{3} r,
\end{array}\right\}
$$

are, respectively, the electric dipole and magnetic dipole operators associated with the atomic current operator $j_{\mu}=(\rho, \mathbf{j})$. E and $\mathbf{B}$ are the induction electric field and magnetic field given (in the Coulomb gauge) in terms of the Heisenberg vector potential field operator $\mathbf{A}(\mathbf{r}, t)$ by :

$$
\mathbf{E}(\mathbf{r}, t)=-\frac{\partial \mathbf{A}(\mathbf{r}, t)}{\partial t}, \quad \mathbf{B}=\operatorname{rot} \mathbf{A} .
$$

The expansion of the vector potential in terms of creation and annihilation operators reads :

$$
\begin{aligned}
\mathbf{A}(\mathbf{r}, t)=\left(\frac{\hbar}{\varepsilon_{0} L^{3}}\right)^{1 / 2} \sum_{\lambda, \mathbf{k}} \frac{\boldsymbol{\varepsilon}_{\lambda, \mathbf{k}}}{\sqrt{2 \omega}} \times \\
\times\left(\mathrm{e}^{i \mathbf{k} . \mathbf{r}-i \omega t} a_{\lambda \mathbf{k}}+\mathrm{e}^{-i \mathbf{k} . \mathbf{r}+i \omega t} a_{\lambda \mathbf{k}}^{+}\right),
\end{aligned}
$$

where the operators $a_{\lambda \mathbf{k}}$ and $a_{\lambda \mathbf{k}}^{\dagger}$ respectively annihilate and create photons of impulsion $\hbar \mathbf{k}$ and transverse polarization states $\varepsilon_{\lambda}(\lambda=1,2)$ localized within a volume $L^{3}$. In $H_{1}^{\mathrm{em}}, \mathbf{E}$ and $\mathbf{B}$ are taken at $t=0$ and evaluated at the centre of mass of the atom $(\mathbf{r}=0)$. In terms of $a_{\lambda \mathbf{k}}$ and $a_{\lambda \mathbf{k}}^{\dagger}, H_{1}^{\mathrm{em}}$ takes the simple form :

$$
\begin{array}{r}
H_{1}^{\mathrm{em}}=\left(\frac{\hbar}{\varepsilon_{0} L^{3}}\right)^{1 / 2} \sum_{\lambda, k} \frac{-i}{\sqrt{2 \omega}}(\omega \mathbf{d}-\mathbf{k} \wedge \boldsymbol{\mu}) . \varepsilon_{\lambda \mathbf{k}} \\
\times\left(a_{\lambda, \mathbf{k}}-a_{\lambda, \mathbf{k}}^{\dagger}\right) .
\end{array}
$$

Let us look at the photons emitted along the $z$ direction with a circular polarization $\xi= \pm 1$ described by the complex polarization vector

$$
\boldsymbol{\varepsilon}_{\xi}=\frac{1}{\sqrt{2}}(\hat{x}+\xi i \hat{y}),
$$

where $\hat{x}$ and $\hat{y}$ are unit vectors along the $x$-and $y$-axes.

The emission probability $p(\xi)$ is proportional to the quantity :

$$
\left|\langle\mathrm{f}|\mathbf{d}| \mathrm{i}\rangle \boldsymbol{\varepsilon}_{\xi}^{*}-\hat{z} \wedge\left\langle\mathrm{f}\left|\frac{\boldsymbol{\mu}}{c}\right| \mathrm{i}\right\rangle \cdot \boldsymbol{\varepsilon}_{\xi}^{*}\right|^{2},
$$

where $|i\rangle$ and $|f\rangle$ are, respectively, the initial and final atomic states involved in the transition. Introducing now the spherical components $d_{q}$ and $\mu_{q}$ of 
the operators $\mathbf{d}$ and $\boldsymbol{\mu}$ :

$$
\left.\begin{array}{l}
d_{ \pm 1}=\mp \frac{1}{\sqrt{2}}\left(d_{x} \pm i d_{y}\right), \\
\mu_{ \pm 1}=\mp \frac{1}{\sqrt{2}}\left(\mu_{x} \pm i \mu_{y}\right),
\end{array}\right\}
$$

we can write :

$$
\boldsymbol{\varepsilon}_{ \pm}^{*} \cdot \mathbf{d}= \pm d_{\mp 1} ; \quad\left(\hat{z} \wedge \varepsilon_{ \pm}^{*}\right) \cdot \boldsymbol{\mu}=i \mu_{\mp 1} .
$$

Using the Wigner-Eckart theorem, we define the reduced $E_{1}$ and $M_{1}$ amplitudes as :

$$
\left.\begin{array}{l}
\left\langle\mathrm{f}\left|d_{q}\right| \mathrm{i}\right\rangle=\frac{(-1)^{J_{\mathrm{f}}-J_{\mathrm{i}}-1}}{\left(2 J_{\mathrm{f}}+1\right)^{1 / 2}}\left\langle J_{\mathrm{f}} M_{\mathrm{f}} \mid 1 q J_{\mathrm{i}} M_{\mathrm{i}}\right\rangle E_{1} \\
\left\langle\mathrm{f}\left|\frac{\mu_{q}}{c}\right| \mathrm{i}\right\rangle=\frac{(-1)^{J_{\mathrm{f}}-J_{\mathrm{i}}-1}}{\left(2 J_{\mathrm{f}}+1\right)^{1 / 2}}\left\langle J_{\mathrm{f}} M_{\mathrm{f}} \mid 1 q J_{\mathrm{i}} M_{\mathrm{i}}\right\rangle M_{1}
\end{array}\right\} .
$$

The circular polarization emitted by atoms initially unpolarized is given by :

$$
P_{\mathrm{c}}=\frac{\sum_{\xi M_{\mathrm{i}} M_{\mathrm{f}}} \xi p(\xi)}{\sum_{\xi M_{\mathrm{i}} M_{\mathrm{f}}} p(\xi)}=\frac{\sum_{M_{\mathrm{i}} M_{\mathrm{f}}}\left(\left|\left\langle\mathrm{f}\left|\left(\mathrm{d}_{-1}+i \mu_{-1}\right)\right| \mathrm{i}\right\rangle\right|^{2}-\left|\left\langle\mathrm{f}\left|\left(-d_{+1}+i \mu_{+1}\right)\right| \mathrm{i}\right\rangle\right|^{2}\right)}{\sum_{M_{\mathrm{i}} M_{\mathrm{f}}}\left(\left|\left\langle\mathrm{f}\left|\left(d_{-1}+i \mu_{-1}\right)\right| \mathrm{i}\right\rangle\right|^{2}+\left|\left\langle\mathrm{f}\left|\left(-d_{+1}+i \mu_{+1}\right)\right| \mathrm{i}\right\rangle\right|^{2}\right)} .
$$

Making use of the identity :

$$
\begin{aligned}
\sum_{M_{\mathbf{i}} M_{\mathbf{f}}}\left|\left\langle J_{\mathrm{f}} M_{\mathrm{f}} \mid 1 q J_{\mathrm{i}} M_{\mathrm{i}}\right\rangle\right|^{2}= \\
=\sum_{M_{\mathbf{i}} M_{\mathrm{f}}}\left|\left\langle J_{\mathrm{f}} M_{\mathrm{f}} \mid 1-q J_{\mathbf{i}} M_{\mathrm{i}}\right\rangle\right|^{2},
\end{aligned}
$$

we obtain the final expression :

$$
\begin{aligned}
P_{\mathrm{c}} & =\frac{\left|E_{1}+i M_{1}\right|^{2}-\left|E_{1}-i M_{1}\right|^{2}}{\left|E_{1}+i M_{1}\right|^{2}+\left|E_{1}-i M_{1}\right|^{2}} \\
& =\frac{2 \operatorname{Im}\left(E_{1} M_{1}^{*}\right)}{\left|E_{1}\right|^{2}+\left|M_{1}\right|^{2}} .
\end{aligned}
$$

The product $E_{1} M_{1}^{*}$ is non zero if and only if the atomic states $|\mathrm{i}\rangle$ and $|\mathrm{f}\rangle$ are mixtures of states having different parity. The fact that one observes circularly polarized photons, although the initial conditions are symmetrical, indicates clearly that all orientations of space are not equivalent.

We would like to show now that, if the atomic Hamiltonian, while violating parity, is invariant under time reflexion, the product $E_{1} M_{1}^{*}$ is purely imaginary. Let $T$ be the antiunitary transformation associated with time reflexion. The transformation properties of $\mathbf{d}$ and $\boldsymbol{\mu}$ can be read off from formulae (32), knowing those of the electric four-current :

$$
\begin{aligned}
T \rho T^{-1}=\rho ; & T \mathbf{j} T^{-1}=-\mathbf{j} \\
& \quad \text { implies } T \mathbf{d} T^{-1}=\mathbf{d} ; \quad T \mu T^{-1}=-\mu .
\end{aligned}
$$

Or, in terms of the spherical components (36) :

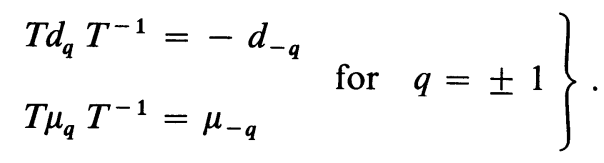

Let now $|\alpha J M\rangle$ be a stationary atomic state ( $\alpha$ stands for a set of quantum numbers invariant under $T$ ) having no other degeneracies than that connected with space rotation invariance. If $T$ commutes with the atomic Hamiltonian, we have :

$$
T|\alpha J M\rangle=\eta_{\alpha J}|\alpha J-M\rangle,
$$

where $\eta_{\alpha J}$ is a phase-factor.

We have now all we need to derive the consequences of $T$ invariance for the phase of the product $E_{1} M_{1}^{*}$.

Using the antiunitary properties of $T$ and eq. (39) and (40), we can write :

$$
\begin{aligned}
& \left\langle J_{\mathrm{f}} M_{\mathrm{f}}\left|d_{q}\right| J_{\mathrm{i}} M_{\mathrm{i}}\right\rangle= \\
& \quad=-\eta_{\mathrm{i}}^{*} \eta_{\mathrm{f}}\left(\left\langle J_{\mathrm{f}}-M_{\mathrm{f}}\left|d_{-q}\right| J_{\mathrm{i}}-M_{\mathrm{i}}\right\rangle\right)^{*},
\end{aligned}
$$

or, in terms of the reduced amplitude $E_{1}$ :

$$
E_{1}=-\eta_{\mathrm{i}}^{*} \eta_{\mathrm{f}}(-1)^{J_{\mathrm{f}}+\mathbf{J}_{\mathbf{i}}-1} E_{1}^{*} .
$$

A similar relation is readily obtained for $M_{1}$ :

$$
M_{1}=\eta_{\mathrm{i}}^{*} \eta_{\mathrm{f}}(-1)^{J_{\mathrm{f}}+J_{\mathbf{i}}-1} M_{1}^{*} .
$$

It follows immediately that :

$$
E_{1} M_{1}^{*}=-E_{1}^{*} M_{1} \text {. }
$$

We can conclude that, if $T$ invariance holds :

$$
\left|P_{\mathrm{c}}\right|=2 \frac{\left|E_{1}\right|\left|M_{1}\right|}{\left|E_{1}\right|^{2}+\left|M_{1}\right|^{2}} .
$$

The above derivation, slightly adapted, can be used to obtain the well known result that a stationary state has an electric dipole moment if, and only if, the invariances under time and space reflexion are simultaneously violated. It is then clear that the parity violating potential we are considering here will not induce any linear Stark effect, which is known to be absent in heavy atoms to excellent precision [20].

In practice, parity mixing can always occur, to a small degree, because of the presence of static electric fields. The amplitude of the parity mixing given by formula (10) will correspond, for the case of Cesium, 
to a static electric field of the order $10^{-4}$ volt $/ \mathrm{cm}$ ! Fortunately, we can show that this parity mixing will not give rise to any circular polarization. Let us call $\varepsilon_{0}$ the static electric field and neglect its variation over distances of the order of the atomic radius, so that the perturbation associated with $\varepsilon_{0}$ is just $-\varepsilon_{0} . d$, where $\varepsilon_{0}$ can be considered as a constant field. Let us assume now that we look for circularly polarized photons $\xi_{ \pm}$emitted along an arbitrary direction specified by the unit vector $n$. Let us now consider the operator $\mathcal{R}$, defined as reflexion with respect to the plane containing the origin and parallel to the two vectors $\varepsilon_{0}$ and $\mathbf{n}$. $\mathcal{R}$ changes right circular polarization into left (and vice versa). The perturbation $-\varepsilon_{0} . \mathbf{d}$ is clearly invariant under $R$, while the potential given in eq. (1) is not. If, under initial symmetric conditions, the photon is emitted along $\mathbf{n}$ with a non zero circular polarization, the symmetry under $\mathcal{R}$ is violated. It follows then that the parity mixing produced by static electric fields does not induce any circular polarization.

With a similar argument, one can show that a static magnetic field, although it does not mix states of different parity, can give rise to a circular polarization. In the case of the $6^{2} S_{1 / 2}-7^{2} S_{1 / 2}$ transition of Cesium, which will be discussed below, through a mixing between different hyperfine levels, a field of $0.1 \mathrm{G}$ can produce a difference, between absorption cross-sections for left and right photons, of the order of $10^{-4}$. However, the circular polarization associated with true parity violation will not be affected by a reversal of the direction of propagation of the light with respect to the magnetic field, while the circular polarization induced by the magnetic field will change its sign.

4. Order of magnitude estimate of parity violation effects in atomic transitions. - As an introduction to the more experimental considerations to be given in the following sections, we shall give here an order of magnitude estimate of parity violating effects. Let us write the reduced magnetic dipole matrix element $M_{1}$ as :

$$
M_{1}=\frac{1}{c} \mu_{\mathrm{B}} \mathcal{M}_{1}=\frac{e \hbar}{2 m_{\mathrm{e}} c} \mathcal{M}_{1},
$$

$\mathcal{N}_{1}$ being a dimensionless number, which will be of the order of unity for the normal magnetic dipole transitions, such as those occurring between the lower states of the $\mathrm{Pb}$ atom, for example

$$
\left(6 p^{2}\right)^{1} S_{0} \rightarrow\left(6 p^{2}\right)^{3} P_{1}
$$

For reasons which will become obvious in a moment, we shall also be concerned with twice forbidden magnetic dipole transitions similar to the ${ }^{3} \mathrm{~S}_{1} \rightarrow{ }^{1} \mathrm{~S}_{0}$ transition in Helium which has been observed recently [21]. In such cases $\mathcal{M}_{1}$ is of the order of $10^{-5}$ to $10^{-4}$. To give an estimate of $E_{1}$, we shall make the simplifying assumption that there is only one mixed state of opposite parity (in the more precise discussion given below for the particular case of
Cesium, we have in fact considered mixing with an infinite number of states, including the continuum).

We write $E_{1}$ as given by first order perturbation theory :

$$
E_{1}=\frac{\left\langle n^{\prime} \mathbf{P}_{1 / 2}\left|V_{\text {p.v. }}^{\text {e.N. }}\right| n \mathrm{~S}_{1 / 2}\right\rangle}{\frac{e^{2}}{2 a_{0}}\left(\varepsilon_{n}-\varepsilon_{n^{\prime}}\right)}\left(e a_{0} \varepsilon_{1}\right)
$$

where $E_{1}^{\prime}=e a_{0} \varepsilon_{1}$ is the reduced electric dipole matrix element associated with the allowed transition between the state $\left|n^{\prime} \mathrm{p}_{1 / 2}\right\rangle$ and a state $\left|n^{\prime \prime} \mathrm{S}_{1 / 2}\right\rangle$ of opposite parity. $\varepsilon_{1}$ is a dimensionless number of the order of unity. Using eq. (29) and neglecting factors close to unity, we can write, after some algebric manipulation :

$$
\left|P_{\mathrm{c}}\right|=2\left|\frac{\varepsilon_{1}}{\mathcal{M}_{1}}\right|
$$

as :

$$
\begin{aligned}
\left|P_{\mathrm{c}}\right| \simeq \frac{2}{\pi} & \left(\frac{G_{\mathrm{F}}}{\sqrt{2}} \frac{m_{\mathrm{e}}^{2} c}{\hbar^{3}}\right) \times \\
& \times Z^{2} \alpha\left|Q_{\mathrm{W}}(Z, N)\right| \frac{\left(\varepsilon_{n} \varepsilon_{n^{\prime}}\right)^{3 / 4}}{\left|\varepsilon_{n}-\varepsilon_{n^{\prime}}\right|} K_{\mathrm{r}}\left|\frac{\varepsilon_{1}}{\mathcal{M}_{1}}\right| .
\end{aligned}
$$

All the factors appearing in this formula are dimensionless. Inserting the numerical values

$$
G_{\mathrm{F}} \frac{m_{\mathrm{e}}^{2} c}{\hbar^{3}} \simeq 3 \times 10^{-12} \text { and } \alpha \simeq 1 / 137,
$$

one obtains the final expression, useful for obtaining rapid estimates of $\left|P_{\mathrm{c}}\right|$ :

$$
\begin{aligned}
\left|P_{\mathrm{c}}\right| \simeq 10^{-14} Z^{2}\left|Q_{\mathrm{W}}(Z, N)\right| \times & \\
& \times K_{\mathrm{r}} \frac{\left(\varepsilon_{n} \varepsilon_{n^{\prime}}\right)^{3 / 4}}{\left|\varepsilon_{n}-\varepsilon_{n^{\prime}}\right|}\left|\frac{\varepsilon_{1}}{\mathcal{M}_{1}}\right| .
\end{aligned}
$$

For instance, if one takes :

$$
\left|\frac{\varepsilon_{1}}{\mathcal{M}_{1}}\right| \sim 1, \frac{\left(\varepsilon_{n} \varepsilon_{n^{\prime}}\right)^{3 / 4}}{\left|\varepsilon_{n}-\varepsilon_{n^{\prime}}\right|} \sim 1, \quad\left|Q_{\mathrm{w}}(Z, N)\right| \sim Z,
$$

one finds :

$$
\left|P_{\mathrm{c}}\right| \sim 10^{-14} Z^{3} K_{\mathrm{r}}
$$

For light atoms $\left(Z \leqslant 10, K_{\mathrm{r}} \approx 1\right)$, the expected effects seem discouragingly small. For heavy atoms, with $Z>50$, the factor $Z^{3} K_{\mathrm{r}}$ gives an enhancement of the order of $10^{6}$ or greater. If one takes for instance the case of Lead, where $Z^{3} K_{\mathrm{r}} \simeq 5 \times 10^{6}$, the above simple estimate (formula 45) gives $\left|P_{\mathrm{c}}\right| \sim 0.5 \times 10^{-7}$. Such an effect will still be very hard to detect, but the situation can be seen as becoming comparable to that of nuclear physics if one remembers that the efficiency of circular $\gamma$-ray analysers does not exceed a few percent and that a polarization of $5 \times 10^{-6}$ has been detected in $\mathrm{Ta}^{181}$ [19]. Possible ways of improving the situation will be discussed in section 6.

5. Review of previous attempts to detect parity mixing in atomic states. - In recent years, experiments have been performed in order to put an upper 
limit on the parity mixing amplitude in atomic states. We would like to confront the results of these experiments with the theoretical considerations developed in the preceding section. Since we are concerned here with an interaction which violates parity but preserves time reflexion invariances, we shall not discuss the very beautiful experiment of Sandars and co-workers [20] which was designed to detect an electric dipole moment on the electron. The effects associated with a non-zero electric dipole moment are characteristic of an interaction which violates both space and time reflexion invariances.

5.1 CiRCULAR DICHROISM IN MOLECULAR OXYGEN $\left(\mathrm{O}^{16}\right)_{2}$ [22]. - The spectral line $(7600 \AA)$ investigated is associated with the transition from the ground state ${ }^{3} \Sigma_{\mathbf{g}}^{-}$to the excited state ${ }^{1} \Sigma_{\mathbf{g}}^{+}$. The analysis of the symmetry properties of the two states shows that the transition is of the magnetic dipole type. The experiment goes in two steps. Step one : a measurement of the absorption coefficient, yielding a value for the magnetic dipole matrix element. Step two : a measurement of the circular polarization $P_{\mathrm{c}}^{\mathrm{tr}}$ of the transmitted light. One expects $P_{c}^{\text {tr }}$ to be the polarization associated with the dipole transition $P_{\mathrm{c}}$ multiplied by the absorption coefficient, $4 \times 10^{-2}$, with, as a consequence, an important loss of sensitivity. From the value of the absorption coefficient one can deduce an order of magnitude for the ratio $\left|\varepsilon_{1} / \mathcal{M}_{1}\right|$, where $\varepsilon_{1}$ and $\mathcal{M}_{1}$ are in units $e a_{0}$ and $\mu_{\mathrm{B}} / c$, respectively :

$$
\frac{\varepsilon_{1}}{\mathcal{M}_{1}}=10^{4} \frac{\mu_{\mathrm{B}}}{c e a_{0}}=\frac{\alpha}{2} 10^{4} \simeq 36 .
$$

In table I, we have compared the upper limit obtained by the authors for the value of $P_{\mathrm{c}}$ with the order of magnitude of the effect predicted from formula (44), taking the above value of $\left|\varepsilon_{1} / \mathcal{H}_{1}\right|$ and the factor $\left(\varepsilon_{n} \varepsilon_{n^{\prime}}\right)^{3 / 4} /\left|\varepsilon_{n}-\varepsilon_{n^{\prime}}\right| \sim 1$. For a precise estimate, a detailed knowledge of the wave function of the molecular states involved would be necessary. The upper limit of $\boldsymbol{P}_{\mathrm{c}}$ quoted by the authors seems to us quite optimistic when one looks at the published data. The value quoted in parenthesis would seem to us more realistic.

5.2 Circular POlarization OF THE PHOTONS , EMITTED IN THE MAGNETIC DIPOLE TRANSITION ${ }^{1} \mathrm{~S}_{0} \rightarrow{ }^{3} \mathrm{P}_{1}(\lambda=4618 \AA) \mathrm{IN} \mathrm{Pb}[23]$. - In this experiment, one looks at the circular polarization of photons emitted spontaneously at a wavelength of $4618 \AA$ from a lead vapour lamp. These photons are associated with the magnetic dipole transition $(6 p)^{2}{ }^{1} S_{0} \rightarrow(6 p)^{2}{ }^{3} P_{1}$. The magnetic dipole transition matrix element has been estimated by Garstang [24]. It leads to a rate of $78 \mathrm{~s}^{-1}$, which is to be compared with the lifetime of $5.75 \times 10^{-9} \mathrm{~s}$ of the odd parity level (6p 7s) ${ }^{3} \mathrm{P}_{1}$ [25]. The corresponding ratio $\mid \varepsilon_{1}\left\langle\mathcal{M}_{1}\right|$ is quoted in table $I$. It differs considerably from the value quoted, without explanation, by the author who suggests a much more suppressed dipole transition (by a factor $10^{2}$ ). The circular polarization detected in the forbidden $4618 \AA$ line is comparable to that observed in other lines associated with allowed electric dipole transitions. Consequently it cannot be associated with a genuine parity violating effect but leads only to an upper limit for such an effect.

$$
\begin{array}{lcccc}
\multicolumn{5}{c}{\text { TABLE I }} \\
& \left|\delta_{1} / \mathscr{M}_{1}\right| & Z^{3} K_{\mathrm{r}} & \left|P_{\mathrm{c}}\right|^{\text {th }} & \left|P_{\mathrm{c}}\right|^{\text {exp }} \\
\left(\mathrm{O}^{16}\right)_{2} & \sim 36 & 5 \times 10^{2} & \sim 10^{-10} & \leqslant 6 \times 10^{-4}\left(6 \times 10^{-2}\right) \\
\mathrm{Pb} & \sim 4 & 5 \times 10^{6} & \sim 10^{-7} & \leqslant 5 \times 10^{-4}
\end{array}
$$

(The theoretical numbers are only order of magnitude estimates).

A look at table I shows that the existing limits are rather poor and that effects larger at least by a factor $5 \times 10^{3}$ than those considered in this paper could have escaped observation. The need for more sensitive experiments is evident.

6. A new proposal : induced twice forbidden magnetic transitions (S-S transitions in alkali atoms, etc...). Let us look at the different ways of increasing $P_{\mathrm{c}}$ as given by formula (44). One possibility would be to find a situation where $s$ and $p$ levels are very close to each other. For practical reasons, the ${ }^{2} S_{1 / 2}{ }^{2} P_{1 / 2}$ system of atomic hydrogen seems excluded, at least with the present techniques. One might also consider an $n \mathrm{~S}_{1 / 2}-n \mathrm{P}_{1 / 2}$ in alkali atoms for $\mathrm{n}$ very large; however the ratio

$$
\frac{\left(\varepsilon_{\mathrm{p} n} \varepsilon_{\mathrm{s} n}\right)^{3 / 4}}{\left|\varepsilon_{\mathrm{s} n}-\varepsilon_{\mathrm{p} n}\right|}
$$

appearing in (44) does not increase with $n$; in fact it goes to a finite limit when $n \rightarrow \infty$. In more physical terms, what is gained with a small energy denominator is lost through the decrease of the matrix element of $V_{\text {p.v. }}^{\text {e.N. }}$ arising from the spreading in space of the atomic wave functions when $n \rightarrow \infty$.

Another possibility is to try to increase the factor $\left|\varepsilon_{1} / \mathcal{M}_{1}\right|$ by working with highly forbidden transitions.

The appearance of dye lasers with tunable frequency has opened a new area in atomic spectroscopy and one can contemplate the possibility of exciting twice forbidden magnetic transitions like

$$
(n-1) \mathrm{S}_{1 / 2} \rightarrow n \mathrm{~S}_{1 / 2}
$$

in alkali atoms.

6.1 ThE MAGNETIC DIPOLE AMPLITUdE IN $(n-1) \mathrm{S}_{1 / 2} \rightarrow n \mathrm{~S}_{1 / 2}$ TRANSITIONS IN ALKALI ATOMS. We would like to give here a brief discussion of the mechanisms which can give rise to a non-vanishing transition amplitude between the $\mathrm{S}$ states of alkali atoms.

6.1.1 "Retardation" effect. - This is associated with the phase difference between the waves emitted at different points of the atomic volume and can be described by the following effective magnetic dipole operator $\boldsymbol{\mu}_{\text {ret. }}$ acting on the wave function of the valence $\mathrm{s}$ electron :

$$
\mu_{\text {ret. }}=-\frac{k^{2} r^{2}}{6} \mu_{\mathrm{B}} \sigma
$$


where $r$ is the radial coordinate of the valence electron and $k$ the photon wave number. The matrix element of $r^{2}$ can be estimated by a method used by Bates and Damgaard [26] for the computation of electric dipole amplitudes. For the $6 \mathrm{~S}-7 \mathrm{~S}$ transition, one finds :

$$
-\frac{1}{6} k^{2}\left\langle 7 \mathrm{~S}\left|r^{2}\right| 6 \mathrm{~S}\right\rangle \simeq 0.12 \times 10^{-5} .
$$

6.1.2 Hyperfine mixing. - The hyperfine interaction between the electron spin and the nuclear spin can mix $\mathrm{S}$ states of different radial quantum numbers and give rise to a non-vanishing magnetic dipole amplitude. This effect can be readily estimated by using first order perturbation theory and the FermiSegré formula to compute the matrix element of the hyperfine interaction :

$$
\begin{aligned}
& \frac{\left\langle F^{\prime} M^{\prime}, n S\left|\mu_{\text {h.f. }}\right| F M,(n-1) \mathrm{S}\right\rangle}{\mu_{\mathrm{B}}\left\langle F^{\prime} M^{\prime}|\sigma| F M\right\rangle} \approx \\
& \quad \approx\left(\frac{v_{n-1}}{v_{n}}\right)^{3 / 2} \frac{\left(F-F^{\prime}\right) \Delta W_{n-1}}{E_{n-1}-E_{n}} .
\end{aligned}
$$

where $F=I \pm \frac{1}{2}$ and $F^{\prime}=I \pm \frac{1}{2}$ stand for the total angular momentum of the atom and $I$ for the nuclear spin. $\Delta W_{n-1}$ is the hyperfine splitting in the $(n-1) \mathrm{S}$ state and $E_{n}, E_{n-1}$ the two unperturbed atomic energies. For the $6 S_{1 / 2}-7 S_{1 / 2}$ transition of Cesium, one finds :

$$
\begin{aligned}
\mathcal{M}_{1 \text { h.f. }} & =\frac{\left\langle F^{\prime} M_{\mathrm{F}^{\prime}}, 7 \mathrm{~S}\left|\mu_{\mathrm{h} . \mathrm{f} .}\right| F M_{\mathrm{F}}, 6 \mathrm{~S}\right\rangle}{\mu_{\mathrm{B}}\left\langle F^{\prime} M_{\mathrm{F}^{\prime}}|\sigma| F M_{\mathrm{F}}\right\rangle} \\
& =0.84 \times 10^{-5}\left(F-F^{\prime}\right) .
\end{aligned}
$$

Note the important fact that the magnetic dipole amplitude is proportional to the difference $F-F^{\prime}$. The effects 6.1 .1 and 6.1 .2 have been computed with the same phase convention, namely that of Appendix I.

6.1.3 Relativistic effects. - The mechanisms we have just described do not affect the diagonal part of the magnetic dipole operator. The gyromagnetic ratios of alkali ground states exhibit deviations from those of a free electron. For instance, in the case of Cesium [27] :

$$
\frac{\Delta g}{g_{\mathrm{e}}}=\frac{g_{\mathrm{Cs}}-g_{\mathrm{e}}}{g_{\mathrm{e}}} \simeq 1.1 \times 10^{-4} .
$$

These deviations are usually explained by invoking relativistic corrections. For instance, in the case of Hydrogen-like atoms, these effects can be described by the effective magnetic dipole operator which has to be used with non-relativistic wave functions :

$$
\boldsymbol{\mu}_{\text {rel. }}=\left(\frac{Z e^{2}}{3 m c^{2} r}-\frac{2}{3} \frac{\mathbf{p}^{2}}{m^{2} c^{2}}\right) \mu_{\mathrm{B}} \boldsymbol{\sigma} .
$$

The diagonal and non diagonal matrix elements of $\boldsymbol{\mu}_{\text {rel. }}$ are of the same order of magnitude :

$$
\begin{aligned}
& \left\langle 1 \mathrm{~S}\left|\mu_{\text {rel. } z}\right| 1 \mathrm{~S}\right\rangle=-\frac{1}{3}(Z \alpha)^{2} \mu_{\mathrm{B}}, \\
& \left\langle 2 \mathrm{~S}\left|\mu_{\text {rel. } z}\right| 1 \mathrm{~S}\right\rangle=-(Z \alpha)^{2} \frac{4 \sqrt{2}}{27} \mu_{\mathrm{B}} .
\end{aligned}
$$

Similar expressions describing relativistic corrections to the magnetic dipole operator can be found in the literature [28], [29], [30]. For atomic S states, they can be cast in the following simple form :

$$
\begin{aligned}
\boldsymbol{\mu}_{\mathrm{rel} .}=\mu_{\mathrm{B}}\left\{\sum _ { i = 1 } ^ { i = Z } \boldsymbol { \sigma } _ { i } \left(1-\frac{2}{3}\right.\right. & \left.\frac{\mathbf{p}_{i}^{2}}{m^{2} c^{2}}\right)+ \\
& +\sum_{i=1}^{i=Z} \frac{1}{3 m c^{2}} \frac{Z e^{2}}{r_{i}} \sigma_{i} \\
& \left.-\frac{1}{3 m c^{2}} \sum_{i<j} \frac{e^{2}}{r_{i j}}\left(\sigma_{i}+\sigma_{j}\right)\right\} .
\end{aligned}
$$

In the case of ${ }^{3} S_{1} \rightarrow{ }^{1} S_{0}$ transitions in a helium-like two-electron systems the two-particle part does not contribute, as has been noted by several authors [28]. If one ignores exchange contributions between the valence $s$ electron and the closed-shell electrons, the above expression can be transformed into a oneparticle operator which can be used in alkali S states :

$$
\boldsymbol{\mu}_{\mathrm{rel} .}=\mu_{\mathrm{B}} \boldsymbol{\sigma}\left(1-\frac{2}{3} \frac{\mathbf{p}^{2}}{m^{2} c^{2}}-\frac{1}{3} \frac{V}{m c^{2}}\right),
$$

where $V$ is the electrostatic potential acting on the valence electron. If this formula is used to estimate the $\Delta g$ of Cesium, one finds results which tend to be too small and to have the wrong sign (the opposite conclusion reached by Perl [30] is based on approximations which seem to us hard to justify). This result is perhaps not too surprising if one remembers that we have used an expression which contains only the lowest order correction in $(Z \alpha)^{2}$ and that $(Z \alpha)^{2} \approx 0.16$ for Cesium.

Among the possible higher order effects, which are negligible for light atoms but may become important for Cesium, we would like to mention the mixing induced by the combined effects of electrostatic interaction between the electrons and the spin-orbit coupling, of the Cesium $n \mathrm{~S}$ state to states of angular momentum $L=1$ belonging to configurations of the type $(5 \mathrm{p})^{-1} 6 \mathrm{~s} n^{\prime} \mathrm{p}$. (Mixing with $L=0$ states does not affect the gyromagnetic ratio and does not induce any magnetic dipole transition amplitude between different $\mathbf{S}$ states). We write symbolically the perturbed $\mathrm{S}$ states as :

$$
\begin{aligned}
& |\tilde{6} \mathrm{~S}\rangle=a{ }^{2} \mathrm{~S}_{1 / 2}+b^{2} \mathrm{P}_{1 / 2}+c{ }^{4} \mathrm{P}_{1 / 2}, \\
& |\tilde{7} \mathrm{~S}\rangle=a^{\prime}{ }^{2} \mathrm{~S}_{1 / 2}+b^{\prime}{ }^{2} \mathrm{P}_{1 / 2}+c^{\prime}{ }^{4} \mathrm{P}_{1 / 2},
\end{aligned}
$$


the real coefficients $a, b, c, a^{\prime}, b^{\prime}, c^{\prime}$ satisfying the relations :

$$
\begin{aligned}
a^{2}+b^{2}+c^{2}-1 & =a^{\prime 2}+b^{\prime 2}+c^{\prime 2}-1 \\
& =a a^{\prime}+b b^{\prime}+c c^{\prime}=0 .
\end{aligned}
$$

One finds, using the known values of the Landé coefficients $\Delta g$ :

$$
\begin{aligned}
\frac{\Delta g}{g} & =\frac{1}{3}\left(c^{2}-2 b^{2}\right), \\
\left\langle\widetilde{6} \mathrm{~S}\left|\mu_{z}\right| \tilde{7} \mathrm{~S}\right\rangle & =\frac{\mu_{\mathrm{B}}}{3}\left(c c^{\prime}-2 b b^{\prime}\right) .
\end{aligned}
$$

For a given configuration, if one neglects differences in the energy denominator, the $g$-shift and the magnetic dipole amplitude take the simple forms :

$$
\left.\begin{array}{rl}
\frac{\Delta g}{g} & =-\frac{1}{3} \frac{\zeta^{2}}{\Delta E^{4}} F_{0} G_{1} \\
\frac{\left\langle\tilde{7} \mathrm{~S}\left|\mu_{z}\right| \tilde{6} \mathrm{~S}\right\rangle}{\mu_{\mathrm{B}}} & =-\frac{1}{6} \frac{\zeta^{2}}{\Delta E^{2} \Delta E^{\prime 2}}\left(F_{0} G_{1}^{\prime}+F_{0}^{\prime} G_{1}\right) .
\end{array}\right\}
$$

In the above expressions, $\zeta$ is the spin-orbit parameter of the 5p hole; $\Delta E \approx \Delta E^{\prime}$ are the core excitation energies for $6 \mathrm{~S}$ and $7 \mathrm{~S}$, respectively. $F_{0}, G_{1}$ and $F_{0}^{\prime}, G_{1}^{\prime}$ are the Slater direct and exchange radial integrals with arguments $\left(6 s 5 \mathrm{p} ; 6 s n^{\prime} \mathrm{p}\right)$ and $(7 \mathrm{~s} 5 \mathrm{p}$; $6 \mathrm{~s} n^{\prime} \mathrm{p}$ ), respectively. A semi-quantitative analysis due to Philipps [31] indicates that the shift $\Delta g / g$ has probably the correct sign and the right order of magnitude (at least for Cesium). Computations with hydrogenic wave functions give the ratio $F_{0}^{\prime} / F_{0}$ as between $\frac{1}{4}$ and $\frac{1}{2}$. The $5 \mathrm{p}$ state being tightly bound, the exchange integrals $G_{1}$ and $G_{1}^{\prime}$ receive contributions mainly from the internal part of $6 \mathrm{~s}$ and $7 \mathrm{~s}$ wave functions. The function $R_{n \mathrm{~s}}(r) / R_{n \mathrm{~s}}(0)$ is an analytic entire function of the energy $E_{n}$ and is slowly varying when $r$ is such that the potential energy is large compared to $E_{n}$. We expect then the ratio $G_{1}^{\prime} / G_{1}$ to satisfy the approximate equality :

$$
\frac{G_{1}^{\prime}}{G_{1}} \approx \frac{R_{7 \mathrm{~s}}(0)}{R_{6 \mathrm{~s}}(0)} \approx\left(\frac{v_{6 \mathrm{~s}}}{v_{7 \mathrm{~s}}}\right)^{3 / 2} \simeq 0.51 .
$$

The transition amplitude $\left\langle\tilde{7} S\left|\mu_{z}\right| \tilde{6} S\right\rangle$, in units of $\mu_{\mathrm{B}}$, given by (48) is probably of the same order of magnitude as $\Delta g / g$, but reduced by a factor of the order of $\frac{1}{3}$ to $\frac{1}{2}$.

To summarize the above discussion, we would like to stress several points. i) The hyperfine mixing effect provides us with a lower limit of the order of $10^{-5}$ for the reduced magnetic dipole transition amplitude $\mathcal{M}_{1}$, since an exact accidental cancellation with the contribution from relativistic corrections, cannot occur simultaneously in the $F=I-\frac{1}{2} \rightarrow F^{\prime}=I+\frac{1}{2}$ and $F=I+\frac{1}{2} \rightarrow F^{\prime}=I-\frac{1}{2}$ transitions. ii) Because of the change of sign of the hyperfine mixing contribu- tion, a precise measurement of the ratio of the intensities of these two transitions will offer the possibility of an experimental determination of the relative sign of hyperfine and relativistic contributions. iii) $10^{-4}$ appears as a reasonable upper limit for the reduced dipole transition amplitude $\mathcal{M}_{1}$.

6.2 EXCITATION OF THE $6 \mathrm{~S}_{1 / 2} \rightarrow 7 \mathrm{~S}_{1 / 2}$ TRANSITION IN Cesium. - The twice forbidden magnetic dipole transition in Cesium :

$$
\text { photon }(\lambda=5395 \AA)+6 \mathrm{~S}_{1 / 2} \rightarrow 7 \mathrm{~S}_{1 / 2},
$$

appears for various reasons, to be a good place to look for the effects discussed in this paper. Its principal advantage is the availability of tunable lasers at the right frequency. The experiment will be carried out in two stages ; first one determines the $\left|M_{1}\right|$ amplitude by looking at the intensity $I_{\mathrm{f}}$ of fluorescence light corresponding to the $7 \mathrm{~S}_{1 / 2} \rightarrow 7 \mathrm{P}_{1 / 2}$ transition ; secondly one searches for a dependence of the $6 \mathrm{~S}_{1 / 2} \rightarrow 7 \mathrm{~S}_{1 / 2}$ transition probability upon the state of circular polarization of the incident photons, through a difference between the associated fluorescence yields $I_{\text {right }}$ and $I_{\text {left }}$. The asymmetry

$$
\mathcal{A}=\left(I_{\text {right }}-I_{\text {left }}\right)\left(I_{\text {right }}+I_{\text {left }}\right)^{-1}
$$

is clearly equal to the quantity $\boldsymbol{P}_{\mathrm{c}}$ discussed in the previous section. An order of magnitude estimate of $P_{\mathrm{c}}$ can be made using (44). The analysis performed just above indicates that $\left|\varepsilon_{1} / \mathcal{M}_{1}\right| \sim 10^{4}$ and taking $\frac{\left(\varepsilon_{n} \varepsilon_{n^{\prime}}\right)^{3 / 4}}{\left|\varepsilon_{n}-\varepsilon_{n^{\prime}}\right|} \sim 1$, one finds :

$$
\left|P_{\mathrm{c}}\right| \sim 10^{-4} \text {. }
$$

A detailed computation of the electric dipole amplitude $E_{1 z}$ defined as the matrix element of $d_{z}$, has been performed, taking into account the mixing with all possible states (including the continuum) and using the theoretical machinery developed in the previous sections. The Weinberg model with $\sin ^{2} \theta_{\mathrm{W}}=0.35$, leads to the result :

$$
E_{1 z}=i 0.45 \times 10^{-8} \mu_{\mathrm{B}} / c=i 1.65 \times 10^{-11} e a_{0}
$$

with

$$
\left|M_{1 z}\right|=\left|\left\langle 6 \mathrm{~s}\left|\mu_{z}\right| 7 \mathrm{~s}\right\rangle\right| \sim 10^{-4} \mu_{\mathrm{B}},
$$

one recovers the value obtained by the rough estimate based on (44) $:\left|P_{\mathrm{c}}\right| \simeq 10^{-4}$.

The above result implies that the imaginary part of the mixing amplitude of the $6 \mathrm{~S}$ state with the different $\mathbf{P}$ states, produced by the parity violating time reflexion invariant electron-nucleus interaction considered here, is of the order of $10^{-11}$. It is instructive

$\left.{ }^{2}\right)$ The present result is slightly different from the one quoted in ref. [6] because we have now included a correction of the order of $Z^{-2 / 3}$ which was neglected to first approximation in the evaluation of $R_{n 1}^{\prime}(0)$ (see Appendix I, Section 2). 
to compare this figure with the upper limit of $1.4 \times 10^{-12}$ quoted by Sandars et al. for the real part of the mixing amplitude of the same state, which has to be associated with a simultaneous violation of space and time reflexion invariances [20].

One may also consider transitions to higher $n S$ states, with $n>7$. However besides the fact that $P_{\mathrm{c}}$ shows a tendancy to decrease with $n$, the polarisability of a level grows at least like $\left(v_{n}\right)^{4}$ and the $\mathrm{S}$ states with $n>7$ are more affected by external perturbations (static electric field, collisions, etc...) [32].

The results of an analysis of the more (and less) obvious problems one will have to face in order to complete such an experiment with the required precision (counting rates, effects of residual electric and magnetic fields, multiphoton processes, collision induced transitions, etc...) have been given by the authors in a previous publication [6]. The conclusion was that, unless some serious difficulty has been overlooked, there is a reasonable hope of observing the parity violating effects induced by neutral currents. The details of this analysis together with the computation of the electric dipole amplitudes induced by the parity violating electron-nucleus potential, will be given in a forthcoming publication of the authors [32].

6.3 SOME OTHER SUGGESTIONS. - We would like to discuss briefly other possible experiments. They are of considerable theoretical interest, but, with the present techniques, they appear more difficult than the Cesium one, since they require tunable lasers in the near ultra-violet region.

6.3.1 Excitation of the transition $6 \mathrm{P}_{1 / 2} \rightarrow 7 \mathrm{P}_{1 / 2}$ $\left(\lambda_{0}=2927 \AA\right)$ in Thallium. - Even in the presence of the spin-orbit potential, the radial wave functions for $n \mathrm{P}_{1 / 2}$ states remain orthogonal for different $n$, so that the matrix element of the non-relativistic dipole moment between such states is still zero. An analysis similar to that performed in Cs would lead to a value of $M_{1 z}$ in the range $10^{-4}$ to $10^{-5} \mu_{\mathrm{B}}$. The electric dipole amplitude induced by the parity violating potential, $E_{1 z}$, is expected to be 10 times larger than in the Cesium case, because of the factor $Z^{2} Q_{\mathrm{w}}(Z, N) K_{\mathrm{r}}$. The theoretical interest of the Thallium experiment lies in the fact that, through comparison with the Cesium one, it would give information on the isospin structure of the weak charge $Q_{\mathrm{w}}$.

6.3.2 Excitation of the $\left(6 \mathrm{p}^{2}\right){ }^{3} \mathrm{P}_{0} \rightarrow\left(6 \mathrm{p}^{2}\right){ }^{1} \mathrm{~S}_{0}$ transition in odd neutron lead isotopes $\left(\lambda_{0}=3394 \AA\right)$. In the absence of nuclear spin, the transition ${ }^{3} \mathrm{P}_{0} \rightarrow{ }^{1} \mathrm{~S}_{0}$ is strictly forbidden by conservation of angular momentum, but in Lead isotopes with an odd number of neutrons, the hyperfine interaction will mix the two levels $\left(6 \mathrm{p}^{2}\right){ }^{3} \mathrm{P}_{0}$ and $\left(6 \mathrm{p}^{2}\right){ }^{1} \mathrm{~S}_{0}$ with the level $\left(6 \mathrm{p}^{2}\right)$ ${ }^{3} \mathrm{P}_{1}$. By this mechanism, the magnetic dipole matrix operator acquires a non-vanishing matrix element between the perturbed $\left|{ }^{3} \widetilde{\mathrm{P}}_{0}\right\rangle$ and $\left|{ }^{3} \widetilde{\mathrm{S}}_{0}\right\rangle$ states. The analysis of Margerie [33] concerning the gyromagnetic ratios of these two levels can be readily adapted in order to give a value for the magnetic dipole transition amplitude :

$$
M_{1 z}=\left\langle{ }^{3} \tilde{\mathrm{P}}_{0}\left|\mu_{z}\right|{ }^{3} \widetilde{\mathrm{S}}_{0}\right\rangle=(0.69 \text { to } 0.27) 10^{-6} \mu_{\mathrm{B}} \text {. }
$$

The parity violating potential involving the nuclear spin, which leads to negligible effects in the previous experiments, plays here the dominant role. Through a mixing of the $\left(6 \mathrm{p}^{2}\right){ }^{3} \mathrm{~S}_{0}$ state with the odd parity state $(6 \mathrm{p} 7 \mathrm{~s}){ }^{3} \mathrm{P}_{1}$, it will give rise to a finite electric dipole amplitude : $E_{1 z}=\left\langle{ }^{3} \widetilde{\mathrm{P}}_{0}\left|d_{z}\right|{ }^{3} \widetilde{\mathrm{S}}_{0}\right\rangle$. Although an accurate determination of $E_{1 z}$ for a given parity violating potential would require a precise knowledge of Lead atomic wave functions, an order of magnitude estimate can be obtained from (44) by dropping the factor $Q_{\mathrm{W}}$ and taking $\left|\mathcal{E}_{1} / \mathcal{M}_{1}\right| \sim 10^{6}$. By doing so, one finds :

$$
\left|P_{\mathrm{c}}\right|=2\left|\frac{E_{1 z}}{M_{1 z}}\right| \sim 6 \times 10^{-4} .
$$

We are perfectly aware that the observation of such a highly forbidden transition is a very difficult enterprise, but we believe it is worth consideration for the following two reasons : $\alpha$ ) it will give information on the axial hadronic current which contributes only to the nuclear spin dependent part of the weak electronnucleus potential. $\beta$ ) in some renormalizable models of weak interactions [34], the weak charge $Q_{\mathrm{w}}$ appearing in (1) is identically zero and parity violation arises only through the spin dependent weak potential. In such models, parity violation effects in Cesium will be reduced by a factor $10^{-2}$ and the kind of experiments discussed here would remain the only hope of detecting appreciable effects. A more detailed analysis will be given in a forthcoming publication.

7. Neutral currents in muonic atoms. - In this section, we would like to apply our general analysis to the case of muonic atoms. Because of the size effect, one expects naively that the parity violating effects induced by neutral currents should be larger than in ordinary atoms, and consequently much easier to detect. A more careful analysis shows that this is a highly optimistic view. The main difficulty is a practical one : because of the muon instability, it is not possible to build a target of $\mu$-atoms. Only spontaneous radiative transitions are then accessible to experiment. In order to enhance the parity violating effect, one would like to work with forbidden transitions, but the problem of having an acceptable branching ratio arises immediately. The $\mu$-atoms can be considered to an excellent approximation as hydrogenic atoms, but the nuclear finite size gives rise to substantial energy shifts.

The only possible place to look for parity violating effects appears to be the spontaneous $2 \mathrm{~S}_{1 / 2} \rightarrow 1 \mathrm{~S}_{1 / 2}$ magnetic dipole transition. A simple estimate of the circular polarization $P_{\mathrm{c}}$, valid for $Z \lesssim 20$, can be obtained from formulae (7), (46) and (47), if one uses 
hydrogenic wave functions (with no nuclear size correction) :

$$
P_{\mathrm{c}}=-\frac{Z \alpha}{3 \pi} \cdot \frac{G_{\mathrm{F}}}{\sqrt{2}} \frac{m_{\mu}^{2} c}{\hbar^{3}} \cdot \frac{m_{\mu} c^{2}}{\Delta E} Q_{\mathrm{w}}(Z, N) .
$$

In this formula, $m_{\mu}$ is the muon mass and $\Delta E$ the energy difference between the $2 \mathrm{~S}_{1 / 2}$ and $2 \mathrm{P}_{1 / 2}$ states which, for $Z \geqslant 6$, is due almost entirely to the nuclear size effect. If the radius of the $2 \mathrm{~s}$ Bohr orbit is large compared to the nuclear radius, i. e. if $Z \lesssim 20$, a fair estimate of $\Delta E$ can be obtained from the simple formula :

$$
\Delta E \approx \frac{1}{20} m_{\mu} c^{2}(Z \alpha)^{2}\left(\frac{Z R}{a_{\mu}}\right)^{2} .
$$

where $a_{\mu}=\hbar / m_{\mu} \alpha c$ is the first Bohr orbit radius for the muonic atom and $R$ the nuclear radius, given approximately by :

$$
R \simeq r_{0} A^{1 / 3} \times 10^{-13} \mathrm{~cm} \text { with } r_{0} \sim 1.2-1.3 .
$$

A close inspection of the above formulae shows that $P_{\mathrm{c}}$ goes roughly like

$$
Z \times Q_{\mathrm{w}} \times \frac{1}{Z^{4} A^{2 / 3}} \sim \frac{1}{Z^{2} A^{2 / 3}} .
$$

It is then clear that the largest values of $P_{\mathrm{c}}$ (of the order of $10 \%$ ) are reached for low values of $Z$. Unfortunately, because of the presence of many competing decay processes, the effects one could hope to observe in practice are much smaller. Let us first give the value of the $M_{1}$ decay rate calculated with no nuclear size correction [35] :

$$
\Gamma\left(2 \mathrm{~S} \stackrel{M_{1}}{\longrightarrow} 1 \mathrm{~S}+\gamma\right) \simeq 0.5 \times 10^{-3} \mathrm{~s}^{-1} Z^{10} .
$$

a) The most obvious competing process is the two $E_{1}$-photon $2 \mathrm{~S} \rightarrow 1 \mathrm{~S}$ transition. Its rate has been computed by many authors. We quote the latest result, which includes relativistic corrections, but no nuclear size effects [35] :

$$
\Gamma\left(2 \mathrm{~S} \stackrel{2 E_{1}}{\longrightarrow} \gamma+\gamma+1 \mathrm{~S}\right) \simeq 1.6 \times 10^{3} \mathrm{~s}^{-1} Z^{6} .
$$

b) Another radiative process, which is important only for $Z \leqslant 10$, has been considered by Ruderman [36]. For such light nuclei, the energy separation between the $|2 \mathrm{~S}\rangle$ and $|2 \mathrm{P}\rangle$ states is smaller than, or comparable to, the average energy of the atomic electrons surrounding the muon. The muon-electron state $|2 \mathrm{~S}\rangle \otimes\left|\psi_{\mathrm{el}}\right\rangle$ is mixed by the Coulomb muon-electron interaction with states $|2 \mathrm{P}\rangle \otimes\left|\psi_{\mathrm{el}}^{*}\right\rangle$, where the electronic states $\left|\psi_{\mathrm{el}}\right\rangle$ and $\left|\psi_{\mathrm{el}}^{*}\right\rangle$ have opposite parity. Allowed $E_{1}$ transition to states $|1 \mathrm{~S}\rangle \otimes\left|\psi_{\mathrm{el}}^{*}\right\rangle$ is then possible. With the help of a sum rule, Ruderman has found a compact formula for the rate, summed upon all the electronic final states. In a Hartree approximation for $\left|\psi_{\mathrm{e} 1}\right\rangle$, it reduces to :

$$
\begin{aligned}
\Gamma(2 \mathrm{~S} \stackrel{\text { mixing }}{\longrightarrow} 1 \mathrm{~S}+ & \gamma)=3 \times 10^{6} \mathrm{~s}^{-1} \times \\
& \times \frac{Z^{2}}{Z-1} \sum_{i}\left\langle\psi_{\mathrm{el}}\left|\frac{a_{0}}{r_{i}}\right| \psi_{\mathrm{el}}\right\rangle,
\end{aligned}
$$

where $r_{i}$ is the distance of the $i$ th electron of the cloud from the nucleus. A rough estimate of the atomic matrix element gives values varying from $10^{7} \mathrm{~s}^{-1}$ to $10^{8} \mathrm{~s}^{-1}$ for $2 \leqslant Z \leqslant 6$. The photons associated with this transition have an energy slightly lower than those coming through the direct $M_{1}$ transition, but in view of the energy resolution usually reached in muonic X-ray spectroscopy, a separation of the two kinds of photons would seem very difficult to achieve. Leading to different final atomic states, the two processes do not interfere. If the separation is not possible, any parity violating effects occurring in the forbidden $M_{1}$ transition (photon polarization, etc...) will be effectively reduced by a factor $10^{4}-10^{6}$ since an $E_{1}$ allowed transition is practically unaffected by the parity mixing considered here. For increasing $Z(Z>10)$, the finite nuclear size effects make the energy difference $E_{2 \mathrm{~s}}-E_{2 \mathrm{P}}$ become larger than the electronic energy. The mixing mechanism is then much less efficient and, for $Z>20$, it becomes smaller than the $M_{1}$ one-photon decay rate.

c) The 2P level being less affected than the $2 \mathrm{~S}$ by the finite size of the nucleus, the $2 S$ lies above the $2 \mathrm{P}$ for $Z \geqslant 6$, and the radiative $E_{1}$ transition, $2 \mathrm{~S} \rightarrow 2 \mathrm{P}+$ photon, becomes energetically possible. An order of magnitude estimate can be obtained using the approximate expression for $\Delta E$ given by (50) :

$$
\Gamma\left(2 \mathrm{~S} \stackrel{E_{1}}{\longrightarrow} 2 \mathrm{P}+\gamma\right) \sim 10^{-4} A^{2} Z^{10} \mathrm{~s}^{-1} .
$$

d) Non-radiative decays of the Auger type are very important for $Z<20$. There is first the $2 S \rightarrow 1 S$ Auger transition, where a longitudinal $E_{0}$ photon is exchanged between the muon and an electron. This rate computed by Ruderman [36] has a very slow variation with $Z$ :

$$
\Gamma(2 \mathrm{~S} \stackrel{\text { Auger }}{\longrightarrow} 1 \mathrm{~S}) \sim 2 \times 10^{9} \mathrm{~s}^{-1} .
$$

When the $2 \mathrm{~S}$ becomes higher than the $2 \mathrm{P}$ level, the Auger $2 \mathrm{~S} \rightarrow 2 \mathrm{P}$ transitions become a very efficient desexcitation mechanism and dominate the $2 \mathrm{~S} \rightarrow 2 \mathrm{P}$ radiative transition for $Z \lesssim 20$. The rate, which can be estimated using the internal conversion coefficient of nuclear spectroscopy [37], again varies slowly with $Z$ :

$$
\Gamma(2 \mathrm{~S} \stackrel{\text { Auger }}{\longrightarrow} 2 \mathrm{P}) \sim 10^{13} \mathrm{~s}^{-1} \quad(Z \sim 10-20) .
$$


What conclusions can one draw from the above analysis ? First, the region with $Z \lesssim 10$ is excluded because of the importance of the radiative $2 S \rightarrow 1 S$ decay induced by the $2 \mathrm{P} \rightarrow 2 \mathrm{~S}$ mixing (produced by interaction of the muon with the electronic cloud). Secondly, the very low branching ratio $\left(10^{-6}-10^{-5}\right)$, due to the importance of non radiative decay processes, is an obstacle which is almost impossible to overcome for $10 \lesssim Z<20$.

The situation looks better for $20<Z<40$. As an illustration, let us consider the results concerning $\mathrm{Ti}^{22}$, where the observation of weak $n P \rightarrow 2 \mathrm{~S}$ transitions has allowed a determination of the 2S-2P splitting : $\Delta E=(11 \pm 1) \mathrm{keV}$ [38]. Inserting this value in formula (49), one obtains, in the framework of the Weinberg model with $\sin ^{2} \theta_{\mathrm{w}}=0.35$ :

$$
P_{\mathrm{c}} \sim 0.5 \times 10^{-3} \text {. }
$$

This number will be reduced by about ten per cent because of the finite nuclear size effects not included in (49).

The non radiative $2 S \rightarrow 1 S$ Auger transition will still dominate, leading to a branching ratio for the $M_{1}$ mode of the order of $0.5 \times 10^{-3}$. By going to slightly higher values of $Z$, one improves the branching ratio but at the price of losing on the value of $P_{\mathrm{c}}$. For high values of $Z(Z>40)$, both the circular polarization $P_{\mathrm{c}}$ and the branching ratio are reduced.

In the cascade process, following the capture of a muon by an atom, the probability of passing through the $2 \mathrm{~S}$ state can be shown theoretically to be only of the order of a few per cent. This fact has been verified experimentally on $\mathrm{Ti}^{22}$, where the lines $n \mathrm{P} \rightarrow 2 \mathrm{~S}$ are much less intense than the lines $n^{\prime} \mathrm{S} \rightarrow 2 \mathrm{P}$ and $n^{\prime \prime} \mathrm{D} \rightarrow 2 \mathrm{P}$. Thus, if $N$ is the number of muons stopped per second in $\mathrm{Ti}$, the number of observed $2 \mathrm{~S} \stackrel{M_{1}}{\longrightarrow} 1 \mathrm{~S}+\gamma$ should be only of the order of $10^{-5} \mathrm{~N}$.

Instead of trying to measure $P_{\mathrm{c}}$ directly, it is better to use the fact that, for spin zero nuclei, the muon keeps an appreciable fraction of its initial polarization (about $15 \%$ ) in the cascade process. The angular distribution of photons should exhibit a forward-backward asymmetry, with respect to the muon polarization $\mathbf{P}_{\mu}$, given by :

$$
I=I_{0}\left(1+a \frac{\mathbf{P}_{\mu} \cdot \mathbf{k}}{\left|\mathbf{P}_{\mu}\right| \cdot|\mathbf{k}|}\right),
$$

( $\mathbf{k}$ is the momentum of the photons). Conservation of angular momentum along $\mathbf{k}$ implies $a=P_{\mathrm{c}}$.

To have a chance of seeing an asymmetry of the order of $\sim 10^{-4}$, one has to detect something like $10^{10} M_{1}$ decays, corresponding to $10^{15}$ stopped muons. In order to keep the measuring time $10^{15} N^{-1}$ within reasonable bounds, experiments will have to wait the intense muon beams which will become available with the new generation of meson factories.

Since completing the work presented on muonic atoms, we have received a reprint of Chen and Feinberg [39] dealing with the subject of this last section. Their analysis, which covers a broad range of values of $Z$, makes use of numerical relativistic muonic wave functions and leads to conclusions similar to ours.

Conclusion. - The short range parity violating electron-nucleus potential associated with the neutral currents produces parity mixing between atomic wave functions. The mixing amplitude in one-valenceelectron atoms can be computed reliably by a remarkably simple formula involving only a knowledge of the energy spectrum. A very striking aspect of the result is a rapid variation with atomic number (faster than $Z^{3}$ ) of the mixing amplitude. Recent progress in laser technology (tunable lasers) offers the possibility of exciting twice forbidden magnetic dipole transitions in heavy atoms, where parity violating effects of the order of $10^{-4}$ are expected.

An experimental investigation on Cesium is presently under way at the E.N.S. Laboratory in Paris.

\section{APPENDIX I}

We would like to give here a derivation of the formula giving the starting coefficient of the normalized radial wave function of a valence electron at the nucleus. Instead of using the radial wave function $R_{n l}$, it is more convenient to use the function $u_{n l}(r)=r R_{n l}(r)$. We shall work in the so-called atomic units system and write the binding energy $E_{n l}$ of the valence electron as :

$$
E_{n l}=\frac{1}{2} \varepsilon_{n} m_{\mathrm{e}} c^{2} \alpha^{2}=-\frac{1}{2 v_{n}^{2}} m_{\mathrm{e}} c^{2} \alpha^{2},
$$

where $v_{n}$ is the effective radial quantum number. The quantum defect $\mu\left(\varepsilon_{n}\right)$ is defined as usual as $v_{n}=n-\mu\left(\varepsilon_{n}\right)$. In terms of the reduced variable $\rho=r / a_{0} \quad\left(a_{0}\right.$ is the Bohr radius) the differential equation obeyed by $u_{n l}(\rho)$ reads :

$$
\frac{\mathrm{d}^{2} u_{n l}}{\mathrm{~d} \rho^{2}}+\left[v(\rho)+\varepsilon_{n}-\frac{l(l+1)}{\rho^{2}}\right] u_{n l}(\rho)=0
$$

$v(\rho)$ is the effective potential acting on the valence electron, with the properties :

$$
\text { and } \left.\quad \begin{array}{rl}
\lim \rho \rightarrow 0(\rho v(\rho)) & =2 Z \\
\rho v(\rho) & =2 \text { for } \rho>\rho_{\mathrm{c}}
\end{array}\right\}
$$

In practice, the latter condition will be satisfied only approximately. $\rho_{\mathrm{c}}$ can be interpreted as the ion core radius. 
We shall first use quantum defect theory [14] [15], to give an expression for $u_{n l}(\rho)$ valid for $\rho>\rho_{\mathrm{c}}$ and involving only the quantum defect $\mu(\varepsilon)$ and its derivative with respect to the energy. Then we shall develop a generalized J.W.K.B. approximation, which will give us an almost exact wave function near the origin. In the next step, we shall show that it is possible to choose the normalization of this approximate wave function in such a way that it coincides with the wave function given by the quantum defect theory fro $\rho>\rho_{\mathrm{c}}$. Proving this identification, which turns out to be quite an arduous task, will be done for the region $\rho \gg 1$, assuming $\left|\varepsilon_{n}\right| \ll 1$. It will lead to a value of the wave function near the origin in which terms of the order of $\varepsilon_{n}^{2}$ and $\varepsilon_{n} / Z^{2}$ are neglected. We shall also consider the effect of the electrostatic potential of the electrons near the origin.

1. The radial wave function in the external region $\rho>\rho_{\mathrm{c}}$ and the quantum defect theory. - We shall first give a short review of the quantum defect theory developed by Ham [14] and Seaton [15]. One introduces a function $U_{l \varepsilon}(\rho)$ which is defined for an arbitrary value of the reduced energy $\varepsilon$ and which behaves at the origin like $\rho^{l+1}$. Ham has proved that, provided certain regularity conditions on $v(\rho)$ are satisfied, and with a proper normalization, the function $U_{l \varepsilon}(\rho)$ is an entire analytic function of $\varepsilon$. When $\rho>\rho_{\mathrm{c}}$, $U_{l \varepsilon}(\rho)$ is a well-defined combination of independent solutions of the Schrödinger equation for a pure Coulomb field, and can be written as follows :

$$
U_{l \varepsilon}(\rho)=y_{1 l}(\varepsilon, \rho)-\beta(\varepsilon) y_{4 l}(\varepsilon, \rho),
$$

where $y_{1 l}(\varepsilon, \rho)$ and $y_{4 l}(\varepsilon, \rho)$ are, in the notation of Seaton, the two Coulomb wave functions which are analytic entire functions of $\varepsilon$. From the Ham theorem, it follows that the coefficient $\beta(\varepsilon)$ is also an entire function of $\varepsilon$. There exists another form of $U_{l \varepsilon}(\rho)$ which, in some cases, is more convenient :

$$
\begin{array}{r}
U_{l \varepsilon}(\rho)=y_{1 l}(\varepsilon, \rho)[1+G(\varepsilon, l) \beta(\varepsilon)]- \\
-\beta(\varepsilon) y_{3 l}(\varepsilon, l),
\end{array}
$$

where $y_{3 l}(\varepsilon, \rho)$ is no longer an analytic entire function of $\varepsilon$, but admits a very simple asymptotic expansion in a power series of $\varepsilon . G(\varepsilon, l)$ is a well-defined function of $\varepsilon$ and $l$ given in reference [15]. When $\varepsilon$ is equal to a particular eigenvalue $\varepsilon_{n}$, the condition $U_{l \varepsilon}(\rho) \rightarrow 0$, when $\rho \rightarrow \infty$, implies the eigenvalue condition :

$\beta\left(\varepsilon_{n}\right)=-\left[A\left(v_{n}, l\right) \operatorname{cotg}\left(\pi v_{n}\right)+G\left(\varepsilon_{n}, l\right)\right]^{-1}$,

with

$$
A\left(v_{n}, l\right)=\frac{\Gamma\left(v_{n}+l+1\right)}{v_{n}^{2 l+1} \Gamma\left(v_{n}-l\right)}
$$

In the argument of the cotangent, one can replace $-v_{n}$ by the quantum defect $\mu_{n}$. The entire function $\beta(\varepsilon)$ is given by the above expression for the infinite sequence $\varepsilon_{1} \ldots \varepsilon_{n}$ and, under some well-defined conditions, $\beta(\varepsilon)$ is then determined by the knowledge of the spectrum. One can thus define an interpolated quantum defect $\mu(\varepsilon)$ by the relation :

$$
\beta(\varepsilon)=[A(v, l) \operatorname{cotg}(\pi \mu(\varepsilon))-G(\varepsilon, l)]^{-1} .
$$

In practice, when one wants to construct the function $\mu(\varepsilon)$ from the spectrum, the interpolation can be performed more reliably using the so-called « $\eta$ » defect of Ham, defined as :

$$
A(v, l) \operatorname{cotg}(\pi \mu(\varepsilon))=\operatorname{cotg}(\pi \eta(\varepsilon)) \text {. }
$$

Note that for $l=0, \mu(\varepsilon)=\eta(\varepsilon)$.

When $\varepsilon=\varepsilon_{n}$, the function $U_{l \varepsilon}(\rho)$ is not normalized to unity. Seaton has shown that the function $u_{n l}(\rho)$, where $\int_{0}^{\infty} u_{n l}^{2}(\rho) \mathrm{d} \rho=1$, is given in terms of $U_{l \varepsilon_{n}}(\rho)$, for $\rho>\rho_{\mathrm{c}}$, by :

with

$$
u_{n l}(\rho)=\left(\Delta\left(\varepsilon_{n}\right)\right)^{-1 / 2} U_{l \varepsilon_{n}}(\rho),
$$

$$
\Delta\left(\varepsilon_{n}\right)=\frac{v_{n}^{3} \beta^{2}\left(\varepsilon_{n}\right) A\left(v_{n} l\right)\left(1+\left.\frac{\partial \mu}{\partial v}\right|_{\varepsilon=\varepsilon_{n}}\right)}{\sin ^{2}\left(\pi \mu\left(\varepsilon_{n}\right)\right)} .
$$

It is also of some interest to write $u_{n l}(\rho)$ in terms of the Whittaker function $W_{v_{n} ; l+1 / 2}\left(2 \rho / v_{n}\right)$ :

$$
\begin{aligned}
u_{n l}(r) & =(-1)^{n+l+1} \times \\
& \times\left[\left(1+\frac{\partial \mu}{\partial v}\right) v_{n}^{2} \Gamma\left(v_{n}-l\right) \Gamma\left(v_{n}+l+1\right)\right]-1 / 2 \\
& \times W_{v_{n} ; l+1 / 2}\left(\frac{2 \rho}{v_{n}}\right)
\end{aligned}
$$

This form has been used by Bates and Damgaard [26] to compute electric dipole matrix elements, assuming that they are dominated by the external part of the wave function $\left(\rho>\rho_{\mathrm{c}}\right)$.

Using (A I.5), (A I.6) and (A I.8), one can write $U_{l \varepsilon}(\rho)$ as :

$$
\begin{aligned}
U_{l \varepsilon}(\rho)= & \frac{\beta(\varepsilon)}{\sin \pi \eta(\varepsilon)} \times \\
& \times\left(y_{1 l}(\varepsilon, \rho) \cos \pi \eta(\varepsilon)-y_{3 l}(\varepsilon, \rho) \sin \pi \eta(\varepsilon)\right) .
\end{aligned}
$$

Introducing $\bar{y}_{3}=A^{-1}(v, l) y_{3}$, and using the relation (A I.8), we obtain an alternative form for $U_{l \varepsilon}(\rho)$ :

$$
\begin{aligned}
U_{l \varepsilon}(\rho)= & \frac{\beta(\varepsilon) A(v, l)}{\sin (\pi \mu(\varepsilon))} \times \\
& \times\left[y_{1 l} \cos \pi \mu(\varepsilon)-\bar{y}_{3 l} \sin \pi \mu(\varepsilon)\right] .
\end{aligned}
$$

For later purposes, we shall need the series expansion, in powers of the energy, of $U_{l \varepsilon}(\rho)$. A convergent series expansion of $y_{1 l}(\varepsilon, l)$ has been derived by Kuhn. It involves Bessel functions $J_{n}(z)$, where the argument $z$ is given in terms of $\rho$ by $: z=\sqrt{8 \rho}$. 
The formula quoted by Seaton [15] gives :

$$
\left.\begin{array}{rl}
y_{1 l}(\varepsilon, \rho) & =y_{1 l}^{(0)}(\rho)+\varepsilon y_{1 l}^{(1)}(\rho)+\cdots \\
y_{1 l}^{(0)} & =\frac{z}{2} J_{2 l+1}(z) \\
y_{1 l}^{(1)}= & \frac{1}{12}\left(\frac{z}{2}\right)^{4} \times \\
& \times\left[J_{2 l+4}(z)-\frac{3}{z}(2 l+2) J_{2 l+3}(z)\right]
\end{array}\right\}
$$

It will appear convenient to express $y_{1 l}^{(1)}$ in terms of $J_{2 l+1}(z)$ and its derivative $J_{2 l+1}^{\prime}(z)$. Using well-known recurrence relations between Bessel functions, an alternative expression for $y_{1 l}^{(1)}$ can be derived :

$$
\begin{aligned}
y_{1 l}^{(1)}=- & \frac{1}{24}\left(\frac{z}{2}\right)^{3} \times \\
& \times\left\{\left[1+\frac{8}{z^{2}} l(l+1)(2 l+1)\right] J_{2 l+1}(z)\right. \\
& \left.-\left[1+\frac{8 l(l+1)}{z^{2}}\right] z J_{2 l+1}^{\prime}(z)\right\} . \quad \text { (A I . }
\end{aligned}
$$

An asymptotic power expansion in $\varepsilon$ for $\bar{y}_{3 l}(\rho, \varepsilon)$ is obtained by the following simple rule : replace in (A I.12) and (A I.13) the Bessel function $J_{n}(z)$ by the Bessel function of the second kind $Y_{n}(z)$.

In the identification process, we shall need expressions for $y_{1 l}^{(\mathrm{i})}(\rho)$ and $\bar{y}_{3 l}^{(\mathrm{i})}(\rho)$, when $\rho \gg 1$. We shall use the well-known expansion of the Bessel functions for large values of the argument :

$$
\begin{aligned}
J_{n}(z)=\left(\frac{2}{\pi z}\right)^{1 / 2} & {\left[P_{n}\left(\frac{1}{z}\right) \cos \left(z-\frac{n \pi}{2}-\frac{\pi}{4}\right)-\right.} \\
& \left.-Q_{n}\left(\frac{1}{z}\right) \sin \left(z-\frac{n \pi}{2}-\frac{\pi}{4}\right)\right] \\
J_{n}^{\prime}(z)=- & \left(\frac{2}{\pi z}\right)^{1 / 2}\left[R_{n}\left(\frac{1}{z}\right) \sin \left(z-\frac{n \pi}{2}-\frac{\pi}{4}\right)+\right. \\
& \left.+S_{n}\left(\frac{1}{z}\right) \cos \left(z-\frac{n \pi}{2}-\frac{\pi}{4}\right)\right],
\end{aligned}
$$

where $P_{n}(1 / z), Q_{n}(1 / z), R_{n}(1 / z), S_{n}(1 / z)$ are asymptotic series in $1 / z$, which can be found in any text-book on special functions. The asymptotic expansions for the Bessel functions of the second kind are readily obtained from the above formula by adding $-\pi / 2$ to the argument of the sine and cosine appearing in the formula. From this, it follows immediately that asymptotic expansions of the linear combinations $(\cos \pi \mu) y_{1 l}^{(\mathrm{i})}(\rho)-(\sin \pi \mu) \bar{y}_{3 l}^{(\mathrm{i})}(\rho)$ can be obtained from the simple rule : perform in the asymptotic formulae of the Bessel functions appearing in (A I.12) or (A I.13) the following replacements :

$$
\cos \left(z-n \frac{\pi}{2}-\frac{\pi}{4}\right) \rightarrow \cos \left(z-n \frac{\pi}{2}-\frac{\pi}{4}+\pi \mu\right) \text {, }
$$

$$
\sin \left(z-n \frac{\pi}{2}-\frac{\pi}{4}\right) \rightarrow \sin \left(z-n \frac{\pi}{2}-\frac{\pi}{4}+\pi \mu\right),
$$

the arguments of the functions $P_{n}, Q_{n}, R_{n}, S_{n}$ remaining unchanged.

Introducing now the function $V_{l \varepsilon}(\rho)$ defined in terms of $U_{l \varepsilon}(\rho)$ by :

$$
U_{l \varepsilon}(\rho)=\frac{\beta(\varepsilon) A(v, l)}{\sin (\pi \mu(\varepsilon))}\left(\frac{z}{2 \pi}\right)^{1 / 2} V_{l \varepsilon}(\rho),
$$

the first two terms of a development of $V_{l \varepsilon}$ as a power series in $\varepsilon$ can be written as asymptotic series in $1 / z$ :

$$
\begin{aligned}
V_{l \varepsilon}(\rho) & =V_{l}^{(0)}(\rho)+\varepsilon V_{l}^{(1)}(\rho), \\
V_{l}^{(0)} & =\cos \alpha_{l} P_{2 l+1}\left(\frac{1}{z}\right)-\sin \alpha_{l} Q_{2 l+1}\left(\frac{1}{z}\right),
\end{aligned}
$$

$$
\begin{aligned}
& V_{l}^{(1)}(z)= \\
& =-\pi \frac{\mathrm{d} \mu}{\mathrm{d} \varepsilon}\left(\sin \alpha_{l} P_{2 l+1}\left(\frac{1}{z}\right)+\cos \alpha_{l} Q_{2 l+1}\left(\frac{1}{z}\right)\right)- \\
& -\frac{z^{2}}{96}\left\{\left[1+\frac{8}{z^{2}} l(l+1)(2 l+1)\right]\right. \\
& \times\left[\cos \alpha_{l} P_{2 l+1}\left(\frac{1}{z}\right)-\sin \alpha_{l} Q_{2 l+1}\left(\frac{1}{z}\right)\right] \\
& +\left[z+\frac{8 l(l+1)}{z}\right] \\
& \left.\times\left[\cos \alpha_{l} S_{2 l+1}\left(\frac{1}{z}\right)+\sin \alpha_{l} R_{2 l+1}\left(\frac{1}{z}\right)\right]\right\},
\end{aligned}
$$

where $\alpha_{l}$ is given by :

$$
\alpha_{l}=z+\pi \mu(0)-(2 l+1) \frac{\pi}{2}-\frac{\pi}{4} .
$$

Although these expressions look rather involved, the rules for obtaining them are rather simple. An important point to note is that $V_{l}^{(1)}(z) / V_{l}^{(0)}(z)$ goes like $z^{3}$ when $1 / z \rightarrow 0$, if one remembers that

and

$$
P_{2 l+1}(0)=R_{2 l+1}(0)=1 \text {, }
$$

$$
Q_{2 l+1}(0)=S_{2 l+1}(0)=0 \text {. }
$$

2. A representation of the wave function in the internal region $\left(\rho<\rho_{\mathrm{c}}\right)$. - We would like to study the function $U_{l \varepsilon}(\rho)$ for $\rho<\rho_{\mathrm{c}}$. For small values of $\rho$, the potential $v(\rho)$ is dominated by the electronnucleon potential $v(\rho) \simeq 2 Z / \rho$; furthermore, the binding energy $\varepsilon$ can be neglected with respect to the potential energy. The wave function should not then be very different from the wave function of a one-electron atom with a nuclear charge $Z$ and zero binding energy, which is given, in terms of the Bessel function of argument $\sqrt{Z} z=\sqrt{8 \rho Z}$, by :

$$
U_{l \varepsilon}(\rho) \approx \text { const. } \times \sqrt{8 Z} J_{2 l+1}(\sqrt{8 Z \rho}) \text {. }
$$


Extending a method originally used by Foldy [11], one is tempted to try for $U_{l \varepsilon}(\rho)$ a representation of the following form :

$U_{l \varepsilon}(\rho)=c_{l}(\varepsilon)\left[\varphi^{\prime}(\rho)\right]^{-1 / 2} \sqrt{8 \varphi(\rho)} J_{2 l+1}(\sqrt{8 \varphi(\rho)})$.

The function $\varphi(\rho)$ obeys the differential equation

$$
\begin{aligned}
\left(\varphi^{\prime}\right)^{2}\left[\frac{2}{\varphi}\right. & \left.-\frac{l(l+1)}{\varphi^{2}}\right]= \\
& =v(\rho)+\varepsilon-\frac{l(l+1)}{\rho^{2}}+\frac{3}{4}\left(\frac{\varphi^{\prime \prime}}{\varphi^{\prime}}\right)^{2}-\frac{1}{2} \frac{\varphi^{\prime \prime \prime}}{\varphi^{\prime}} .
\end{aligned}
$$

For small values of $\rho$, the quantity

$$
D \varphi=\frac{3}{4}\left(\frac{\varphi^{\prime \prime}}{\varphi^{\prime}}\right)^{2}-\frac{1}{2} \frac{\varphi^{\prime \prime \prime}}{\varphi^{\prime}},
$$

involving higher derivatives, is small, since, as we have seen, $\varphi(\rho) \simeq Z \rho$. When the argument of the Bessel function is large enough, one can replace the Bessel function by the first term of its asymptotic expansion, this approximation scheme then reducing to the ordinary J. W. K. B. approximation. In the case of a Coulomb potential, this approximation is known to be valid when the two following conditions can be simultaneously fulfilled : $\rho \varepsilon \leqslant 1, \rho \gg 1$, which is the case provided $\varepsilon$ is sufficiently small. If we are not interested in an evaluation of the quantum defect $\mu$, the fact that $\mathfrak{D} \varphi$ may not be small in an intermediate region $\rho \simeq 1$, will not cause us any trouble.

Let us first consider the region $0 \leqslant \rho \leqslant \rho_{\mathrm{i}}$, where $\rho_{\mathrm{i}}$ is the internal classical turning point. In contrast to the case of the ordinary J. W. K. B. approximation, one can define a zero order approximation $\varphi_{0}(\rho)$, the solution of :

$\varphi^{\prime 2}\left[\frac{2}{\varphi}-\frac{l(l+1)}{\varphi^{2}}\right]=v(\rho)+\varepsilon-\frac{l(l+1)}{\rho^{2}}=Q(\rho)$,

which is regular for $\rho=\rho_{\mathrm{i}}$, by imposing the condition $\varphi_{0}\left(\rho_{\mathrm{i}}\right)=l(l+1) / 2$. One can then easily verify that, if $Q(\rho)$ is indefinitely differentiable for $\rho=\rho_{\mathrm{i}} \varphi_{0}\left(\rho_{\mathrm{i}}\right)$ with $\mathrm{d} Q / \mathrm{d} \rho\left(\rho=\rho_{\mathrm{i}}\right) \neq 0, \varphi_{0}(\rho)$ is also indefinitely differentiable for $\rho=\rho_{\mathrm{i}}$. We shall assume that, for $\rho \leqslant \rho_{\mathrm{i}}, Q(\rho)$ can be approximated by the following expression :

$$
Q(\rho)=\frac{2 Z}{\rho}(1-\zeta Z \rho)-\frac{l(l+1)}{\rho^{2}},
$$

where $\zeta$ is given by :

$$
2 \zeta Z^{2}=\frac{2 V_{\mathrm{e}}}{m_{\mathrm{e}} c^{2} \alpha^{2}}-\varepsilon
$$

$V_{\mathrm{e}}$ being the electrostatic potential of the core electrons. To estimate $\zeta$, we can use the Thomas-Fermi model [40] valid for large values of $Z$, which gives $\zeta=1.80 Z^{-2 / 3}$ (i. e. 0.12 for Cs). For the case of Cs, we also possess numerical values of the potential, chosen so that the binding energies of the lowest 40 levels agree well with experiment [41]. We find that

$$
\begin{aligned}
\frac{2 V_{\mathrm{e}} / m_{\mathrm{e}} c^{2} \alpha^{2}}{2 Z / \rho_{\mathrm{i}}} \approx \zeta \approx & 0.10 \\
& \left(\frac{\varepsilon}{2 Z^{2}} \text { is completely negligible }\right) .
\end{aligned}
$$

The variation $\frac{V_{\mathrm{e}}\left(\rho_{\mathrm{i}}\right)-V_{\mathrm{e}}(0)}{V_{\mathrm{e}}(0)}$ is also small $(\simeq 0.10)$.

It follows that $\zeta$ can be treated as a constant which is small compared to unity. If one takes $\zeta=0$, one obviously gets $\varphi_{0}=Z \rho$. For Cesium, a first order expansion in $\zeta$ is adequate when $\rho \lesssim \rho_{\mathrm{i}}$. We shall write $\varphi_{0}(\rho)$ as :

$$
\varphi_{0}(\rho)=Z \rho\left(1-2 Z^{2} \zeta \chi(\rho)\right) .
$$

To first order in $\zeta$, we have for $\chi^{\prime}(\rho)$ the following equation :

$$
2 \chi^{\prime}(\rho)\left(\rho-\frac{l(l+1)}{2 Z}\right)+\chi(\rho)=\frac{\rho}{2 Z} .
$$

We require that $\chi^{\prime}(\rho)$ should be finite for $\rho=\frac{l(l+1)}{2 Z}$, implying that

$$
\chi\left(\frac{l(l+1)}{2 Z}\right)=\frac{l(l+1)}{4 Z^{2}},
$$

which is equivalent to the condition

$$
\varphi_{0}\left(\rho_{\mathrm{i}}\right)=\frac{l(l+1)}{2}
$$

to first order in $\zeta$. The regular solution is a first order polynomial :

$$
\chi(\rho)=\frac{l(l+1)}{6 Z^{2}}+\frac{\rho}{6 Z} .
$$

We thus obtain, for $\rho \leqslant \rho_{\mathrm{i}}$, and to first order in $\zeta$ :

$\varphi_{0}(\rho)=Z\left(1-\zeta \frac{l(l+1)}{3}\right) \rho-\frac{\zeta}{3} Z^{2} \rho^{2}$.

If we now compute the correction term $D \varphi_{0}$ we find that $D \varphi_{0} / v$ is of the order of $\zeta^{2}$. Hence, to first order in $\zeta$, we have, for $0 \leqslant \rho \leqslant \rho_{\mathrm{i}}$ :

$\varphi(\rho)=Z\left(1-\zeta \frac{l(l+1)}{3}\right) \rho-\frac{\zeta}{3} Z^{2} \rho^{2}+0\left(\zeta^{2}\right)$. 
This result can be applied to the hydrogen atom. In that case, $2 \zeta Z^{2}=-\varepsilon, Z=1$ and to first order in $\varepsilon$, we have :

$\varphi(\rho)=\left(1+\varepsilon \frac{l(l+1)}{6}\right) \rho+\frac{\varepsilon \rho^{2}}{6}+0\left(\varepsilon^{2}\right)$.

Placing this expression in equation (A I.19), one can obtain a first order expansion in $\varepsilon$ of the function $y_{1 l}(\varepsilon, \rho)$, which coincides with the result quoted in (A I. 12).

3. The matching of the internal and external wave functions. - We now turn our attention to the region $\rho_{\mathrm{i}} \leqslant \rho \leqslant \rho_{\mathrm{e}}$, where $\rho_{\mathrm{e}}$ is the external turning point. (See Fig. 2.)

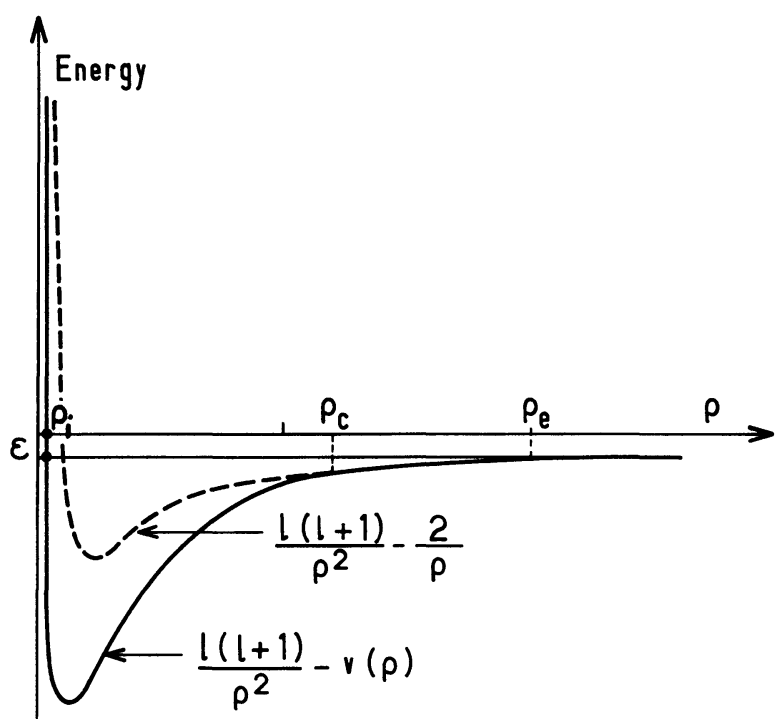

FIG. 2.

For a sufficiently small reduced energy $\varepsilon$, the external turning point is in the Coulomb region, where $v(\rho) \simeq 2 / \rho$.

Then, $\rho_{\mathrm{e}} \simeq-\frac{1}{\varepsilon}-\frac{1}{\varepsilon} \sqrt{1+l(l+1) \varepsilon}$.

When $\rho>\rho_{\mathrm{i}}$, the variation with $\rho$ of the electrostatic potential of the core electrons will become more important and the simplified form (A I.27) is no longer valid, since the corrective term $D \varphi$ may become important. We shall derive an asymptotic series for $\varphi(\rho)$ from an iterative solution of the integral equation obtained by integrating both sides of (A I.20) :

$G(\varphi)=\int_{\rho_{\mathrm{i}}}^{\rho}\left(v(\rho)-\frac{l(l+1)}{\rho^{2}}+\varepsilon+\mathcal{D}\right)^{1 / 2} \mathrm{~d} \rho$,

with

$$
\begin{aligned}
G(\varphi)= & \int_{l(l+1) / 2}^{\varphi}\left[\frac{2}{x}-\frac{l(l+1)}{x^{2}}\right]^{1 / 2} \mathrm{~d} x \\
= & \sqrt{8 \varphi}\left(1-\frac{l(l+1)}{2 \varphi}\right)^{1 / 2}- \\
& -2 \sqrt{l(l+1)} \operatorname{Arcos} \sqrt{\frac{l(l+1)}{2 \varphi}} .
\end{aligned}
$$

For $\rho=\rho_{\mathrm{i}}$, we have $\varphi=l(l+1) / 2$. We shall also assume that $\mathscr{D}\left(\rho_{\mathrm{i}}\right)=0$, since for $\rho \leqslant \rho_{\mathrm{i}}$ this condition is practically verified. In the vicinity of $\rho=\rho_{\mathrm{i}}$, the zero-order approximation is certainly very accurate. When $\rho>\rho_{\mathrm{i}}$, one should keep higher order terms in the iterative procedure. We shall assume that the variations of $v(\rho)$ are sufficiently smooth that, although $\mathscr{D}(\rho)$ may be significantly different from zero for $\rho_{\mathrm{i}}<\rho \ll \rho_{\mathrm{e}}$, it does not produce any new zero of

$$
v(\rho)-\frac{l(l+1)}{\rho^{2}}+\varepsilon-\mathfrak{D} \varphi .
$$

Such zeros are known to appear in the ordinary J. W. K. B. iterative expansion, but thev are connected with the fact that the phase function is singular near $\rho=\rho_{\mathrm{i}}$, while here $\varphi(\rho)$ is regular for $\rho=\rho_{\mathrm{i}}$.

Let us now take $\rho$ such that $\rho>\rho_{\mathrm{c}}$, where $\rho_{\mathrm{c}}$ is the core radius, and where the energy $\varepsilon$ is assumed to be sufficiently small that we can choose $\rho_{\mathrm{c}}$ such that :

$$
1 \ll \rho_{\mathrm{c}} \ll \rho_{\mathrm{e}}(\varepsilon)
$$

The conditions $1 \ll \rho \ll \rho_{\mathrm{e}}(\varepsilon)$ are those required for the validity of the J. W. K. B. approximation for atomic hydrogen, so that we expect an iterative procedure starting with $D \varphi=0$ to be again quite accurate. Using (A I.29), we have :

$$
\begin{aligned}
& G(\varphi)=G\left(\varphi_{\mathrm{c}}\right)+ \\
& +\int_{\rho_{\mathrm{c}}}^{\rho}\left(\frac{2}{\rho}-\frac{l(l+1)}{\rho^{2}}+\varepsilon+D \varphi\right)^{1 / 2} \mathrm{~d} \rho,
\end{aligned}
$$

where $\varphi_{\mathrm{c}}=\varphi\left(\rho_{\mathrm{c}}\right)$.

We write $\varphi=\varphi_{0}+\varphi_{1}$ with

$G\left(\varphi_{0}\right)=G\left(\varphi_{\mathrm{c}}\right)+\int_{\dot{\rho}_{\mathrm{c}}}^{\rho}\left(\frac{2}{\rho}-\frac{l(l+1)}{\rho^{2}}+\varepsilon\right)^{1 / 2}$,

and :

$$
\begin{aligned}
\Delta G & =G\left(\varphi_{0}+\varphi_{1}\right)-G\left(\varphi_{0}\right) \\
& =\frac{1}{2} \int_{\rho_{\mathrm{c}}}^{\rho} \mathrm{d} \rho\left(\frac{2}{\rho}-\frac{l(l+1)}{\rho^{2}}+\varepsilon\right)^{-1 / 2} \mathfrak{D} \varphi_{0} .
\end{aligned}
$$

Next, we shall have to identify the first order expansion in $\varepsilon$ for $\rho>\rho_{\mathrm{c}}$, computed with :

$U_{l \varepsilon}(\rho)=c_{l}(\varepsilon)\left[\varphi^{\prime}(\rho)\right]^{-1 / 2} \sqrt{8 \varphi(\rho)} J_{2 l+1}(\sqrt{8 \varphi(\rho)})$

with that derived from the quantum defect theory given in (A I .18). This identification will be performed in the region of large $\rho$ only, through expansions in 
$1 / z$. We shall push the computation up to the order which is strictly necessary to get $\left.c_{l}\right|_{\varepsilon=0}$ and $\left.\frac{\partial c_{l}}{\partial \varepsilon}\right|_{\varepsilon=0}$. We shall write $\varphi(\rho, \varepsilon)$ as :

$$
\varphi(\rho, \varepsilon)=\varphi^{(0)}(\rho)+\varepsilon \varphi^{(1)}(\rho) .
$$

It will also be convenient to work with the function $\xi(z=\sqrt{8 \rho}, \varepsilon)$ :

$\xi(z, \varepsilon)=\sqrt{8 \varphi(\rho, \varepsilon)}=\xi^{(0)}(z)+\varepsilon \xi^{(1)}(z)+\cdots$.

We shall first give the expression for

$$
\xi_{0}(z, \varepsilon)=\xi_{0}^{(0)}(z)+\varepsilon \xi_{0}^{(1)}(z)
$$

computed with (A I.32), i. e. using the zero order approximation $(\mathfrak{D}=0)$. The results will be presented as an expansion in $1 / z$, keeping only the terms which will be needed later :

$$
\begin{aligned}
& \xi_{0}(z, \varepsilon)=\sqrt{8 \varphi_{0}(\rho, \varepsilon)}=\xi_{0}^{(0)}(z)+\varepsilon \xi_{0}^{(1)}(z)+\cdots \\
&=z+\Lambda^{(0)}+\frac{2 l(l+1) \Lambda^{(0)}}{z\left(z+\Lambda^{(0)}\right)}+ \\
&+\varepsilon\left[\frac{z^{3}}{96}+\frac{l(l+1)}{12} z+\Lambda^{(1)}\right] \\
&\left.+O\left(\frac{1}{z^{4}}\right)+O\left(\frac{\varepsilon}{z}\right) . \quad \text { (A I } .37\right)
\end{aligned}
$$

In this expression $\Lambda^{(0)}$ and $\Lambda^{(1)}$ are numerical constants which can be expressed as integrals involving the ionic potential $v(\rho)$ in the core region. We have, now, to look for corrections arising from the first iteration. Let us first compute the quantity $\mathfrak{D}(\rho, \varepsilon)$ using the above expression for $\xi(\rho, \varepsilon)$ :

$$
\begin{aligned}
& \varphi_{0}(\rho, \varepsilon)=\frac{1}{8}\left(\xi_{0}(\rho, \varepsilon)\right)^{2} \\
&=\rho+\frac{1}{8}\left(\Lambda^{(0)}\right)^{2}+\frac{\sqrt{2}}{2} \Lambda^{0} \sqrt{\rho}+ \\
&+\varepsilon\left(\frac{\rho^{2}}{6}+\frac{\sqrt{2} \Lambda^{(0)} \rho \sqrt{\rho}}{24}+\frac{l(l+1)}{6} \rho\right) \\
&+O\left(\frac{1}{\sqrt{\rho}}\right)+O(\varepsilon \sqrt{\rho}) .
\end{aligned}
$$

(Note that if one takes $\Lambda^{(0)}=0$, one finds the expression (A I.28) for $\varphi(\rho)$ for the case of atomic hydrogen). One obtains :

$$
\begin{aligned}
D \varphi_{0} & =-\frac{3}{32} \sqrt{2} \frac{\Lambda^{(0)}}{\rho^{2} \sqrt{\rho}}+\frac{9}{128} \frac{\left(\Lambda^{(0)}\right)^{2}}{\rho^{3}}- \\
& -\frac{3}{128} \frac{\varepsilon \Lambda^{(0)}}{\rho \sqrt{\rho}}+O\left(\frac{1}{\rho^{3} \sqrt{\rho}}\right)+O\left(\varepsilon \rho^{-1}\right) .
\end{aligned}
$$

The next step is the evaluation of the integral

$$
\begin{array}{r}
\Delta G=\frac{1}{2} \int_{\rho_{\mathrm{c}}}^{\rho}\left(\frac{2}{\rho}-\frac{l(l+1)}{\rho^{2}}+\varepsilon\right)^{-1 / 2} \mathcal{D}\left(\varphi_{0}\right) \\
=\delta^{(0)}+\varepsilon \delta^{(1)}+\frac{3}{8 z}\left[\frac{\Lambda^{(0)}}{z}-\left(\frac{\Lambda^{(0)}}{z}\right)^{2}\right]+ \\
+O\left(\frac{1}{z^{4}}\right)+O\left(\frac{\varepsilon}{z}\right)
\end{array}
$$

where $\delta^{(0)}$ and $\delta^{(1)}$ are constants depending on our particular choice of $\rho_{\mathrm{c}}$. Note that in the integrand of $\Delta G$, the terms in $\varepsilon / \rho$ cancel each other. The correction to $\xi(\rho, \varepsilon)$ can now be readily obtained and can be performed on (A I.37) by the replacement $2 l(l+1) \rightarrow 2 l(l+1)+\frac{3}{8}$ in the term proportional to $\frac{\Lambda^{(0)}}{z\left(z+\Lambda^{0}\right)}$ and by a redefinition of the constants $\Lambda^{(0)}$ and $\Lambda^{(1)}$. This rule remains valid when higher order contributions are considered, up to terms of the order of $1 / z^{4}$ and $\varepsilon / z$, so that the final corrected expression reads :

$$
\begin{aligned}
& \xi(z, \varepsilon)=\sqrt{8 \varphi(\rho, \varepsilon)}=z+\Lambda^{(0)}+ \\
& +\frac{\left[2 l(l+1)+\frac{3}{8}\right] \Lambda^{(0)}}{z\left(z+\Lambda^{(0)}\right)}+O\left(\frac{1}{z^{4}}\right) \\
& +\varepsilon\left[\frac{z^{3}}{96}+\frac{l(l+1) z}{12}+\Lambda^{(1)}\right]+O\left(\frac{\varepsilon}{z}\right) .
\end{aligned}
$$

As we shall see, this expression is accurate enough to allow a determination of $c_{l}(0)$ and $\partial c_{l} / \partial \varepsilon(0)$.

To perform the final step of the identification of the two forms of the solution, it is convenient to rewrite (A I.34) in a slightly different way, by introducing $\xi^{\prime}(z, \varepsilon)=\partial \xi / \partial z$ :

$$
U_{l \varepsilon}(\rho)=2 c_{l}(\varepsilon)\left(\frac{z}{2 \pi}\right)^{1 / 2}\left[\left(\frac{\pi \xi}{2 \xi^{\prime}}\right)^{1 / 2} J_{2 l+1}(\xi)\right]
$$

Defining now the coefficient $D_{l}(\varepsilon)$ as :

$$
c_{l}(\varepsilon)=\frac{1}{2} \frac{\beta(\varepsilon) A(v, l)}{\sin (\pi \mu(\varepsilon))} D_{l}(\varepsilon),
$$

we obtain the following expression for the function $V_{l \varepsilon}(\rho)$, given in terms of $U_{l \varepsilon}$, by (A I.15) :

$$
V_{l \varepsilon}=D_{l}(\varepsilon) \frac{1}{\left(\xi^{\prime}\right)^{1 / 2}}\left(\frac{\pi \xi}{2}\right)^{1 / 2} J_{2 l+1}(\xi)
$$

(One can use this expression as a starting point for a generalized J.W.K.B. expansion. All the results given in this Appendix have also been obtained in this manner.)

Let us consider first the zero order approximation in $\varepsilon$, keeping the dominant term in $z$. From (A I. 17), 
one obtains :

$$
\begin{aligned}
V_{l}^{(0)}=\cos [z+\pi \mu(0)-(2 l+1) & \left.\frac{\pi}{2}-\frac{\pi}{4}\right] \times \\
& \times\left(1+O\left(\frac{1}{z}\right)\right),
\end{aligned}
$$

and from (A I.43) together with (A I.41) :

$$
\begin{aligned}
V_{l}^{(0)}=D_{l}(0) \cos \left[z+\Lambda^{(0)}-(2 l\right. & \left.+1) \frac{\pi}{2}-\frac{\pi}{4}\right] \times \\
& \times\left(1+O\left(\frac{1}{z}\right)\right) .
\end{aligned}
$$

By identification, one finds :

$$
\Lambda^{(0)}=\pi \mu(0)+m \pi, \quad D_{l}(0)=(-1)^{m},
$$

where $m$ is an integer. If one looks at the definition of $\Lambda^{(0)}$, one finds that it is zero when $Z=1, \rho_{\mathrm{c}}=0$ (i. e. for atomic Hydrogen). Since $\mu$ is zero in such a case, one must take $m=0$. We thus conclude that

$$
D_{l}(0)=1, \quad \Lambda^{(0)}=\pi \mu(0) .
$$

Let us now try to identify the two expressions for $V_{l}^{(1)}(\rho)$. By stopping the asymptotic series at the appropriate order, the expression (A I.18) for $V_{l}^{(1)}(\rho)$ can be cast in the following form :

$$
\begin{gathered}
V_{l}^{(1)}(z)=\cos \alpha_{l}\left[a z^{2}+b z+c+O\left(\frac{1}{z}\right)\right]+ \\
+z \sin \alpha_{l}\left[a^{\prime} z^{2}+b^{\prime} z+c^{\prime}+O\left(\frac{1}{z}\right)\right]
\end{gathered}
$$

where $a, b, c, a^{\prime}, b^{\prime}, c^{\prime}$ are constant coefficients which can be readily obtained from the known expressions for the series $P_{2 l+1}\left(\frac{1}{z}\right) \ldots$ We shall put the expression of $V_{l}^{(1)}(z)$ obtained from (A I.43) into a similar form and identify the coefficients $a, b, c, a^{\prime}, b^{\prime}, c^{\prime}$. The interesting quantity $\left.\frac{\partial D_{l}}{\partial \varepsilon}\right|_{\varepsilon=0}$ will appear only in the coefficient $c$. The identification of the five other coefficients will give a good test of the consistency of our procedure. From (A I.43), one has immediately $V_{l}^{(1)}(\rho)$ :

$$
\begin{aligned}
V_{l}^{(1)}(\rho)=\left(\left.\frac{\partial D}{\partial \varepsilon}\right|_{\varepsilon=0}-\frac{1}{2} \frac{\xi^{(1) \prime}}{\xi^{(0) \prime}}+\frac{1}{2} \frac{\xi^{(1)}}{\xi^{(0)}}\right) \times & \\
& \times\left(\frac{\pi \xi^{(0)}}{2 \xi^{(0) \prime}}\right)^{1 / 2} J_{2 l+1}\left(\xi^{(0)}\right) \\
& +\xi^{(1)}\left(\frac{\pi \xi^{(0)}}{2 \xi^{(0) \prime}}\right)^{1 / 2} J_{2 l+1}^{\prime}\left(\xi^{(0)}\right) \\
\xi^{(0)}=\left.\xi\right|_{\varepsilon=0}, \quad \xi^{(0) \prime}=\left.\frac{\partial \xi}{\partial z}\right|_{\varepsilon=0} . & \xi^{(1) \prime}=\left.\frac{\partial^{2} \xi}{\partial \varepsilon \partial z}\right|_{\varepsilon=0} .
\end{aligned}
$$

The explicit calculation of $V_{l}^{(1)}(\rho)$ is straightforward, but one must be careful not to miss terms of the required order. Instead of writing the result exactly in the form (A I.45), we shall use an equivalent form more appropriate for the comparison with the result of the quantum defect theory :

$$
\begin{aligned}
& V_{l}^{(1)}=-\frac{z^{2}}{96}\left\{\left[1-\frac{96}{z^{2}} \frac{\partial D}{\partial \varepsilon}+\dot{O}\left(\frac{1}{z^{3}}\right)\right]\left[\cos \alpha_{l}\left(P_{2 l+1}\left(\frac{1}{z}\right)+O\left(\frac{1}{z^{3}}\right)\right)-\sin \alpha_{l}\left(Q_{2 l+1}\left(\frac{1}{z}\right)+O\left(\frac{1}{z^{2}}\right)\right)\right]+\right. \\
& \left.+\left[z+\frac{8 l(l+1)}{z}+O\left(\frac{1}{z^{2}}\right)\right]\left[\cos \alpha_{l}\left(S_{2 l+1}\left(\frac{1}{z}\right)+O\left(\frac{1}{z^{4}}\right)\right)+\sin \alpha_{l}\left(R_{2 l+1}\left(\frac{1}{z}\right)+O\left(\frac{1}{z^{3}}\right)\right)\right]\right\} \text {. }
\end{aligned}
$$

The coefficients $a, b, c, a^{\prime}, b^{\prime}, c^{\prime}$ can be obtained readily from the above expression. (Note that in (A I.18), the term proportional to $\partial \mu / \partial \varepsilon$, being of high order in $1 / z$, does not contribute to $a, b, c$, $a^{\prime}, b^{\prime}, c^{\prime}$.) By comparing with the expression (A I. 18), one sees immediately that the two sets of coefficients coincide, provided we choose $\left.\frac{\partial D}{\partial \varepsilon}\right|_{\varepsilon=0}$ as follows :

$$
\begin{aligned}
\frac{\partial D}{\partial \varepsilon}=-\frac{8}{96} l & (l+1)(2 l+1)= \\
& =-\frac{1}{12} l(l+1)(2 l+1)=-\frac{1}{2} \sum_{p=0}^{p=l} p^{2} .
\end{aligned}
$$

Remembering that $A(v, l)=\frac{\Gamma(v+l+1)}{v^{2 l+1} \Gamma(v-l)}$ can be written as a polynomial in $\varepsilon=-\frac{1}{v^{2}}$ :

$$
\begin{aligned}
A(v, l) & =\frac{\left(v^{2}-l^{2}\right)\left(v^{2}-(l-1)^{2}\right) \ldots\left(v^{2}-1\right)}{\dot{v}^{2 l}} \\
& =1+\left(\sum_{p=0}^{p=l} p^{2}\right) \varepsilon+\cdots,
\end{aligned}
$$

we can write $D_{l}(\varepsilon)$ and $c_{l}(\varepsilon)$ as follows :

$$
\begin{gathered}
D_{l}(\varepsilon)=A^{-1 / 2}(v, l)+O\left(\varepsilon^{2}\right), \\
c_{l}(\varepsilon)=\frac{1}{2} \frac{\beta(\varepsilon) A^{1 / 2}(v, l)}{\sin (\pi \mu(\varepsilon))}\left(1+O\left(\varepsilon^{2}\right)\right) .
\end{gathered}
$$


4. Starting coefficient of the radial wave function at the origin. Results. - We are now in position to write the normalized wave function $u_{n l}(r)$ :

$$
\begin{aligned}
u_{n l}(\rho)= & \frac{1}{2}\left[v_{n}^{3}\left(1+\left.\frac{\partial \mu}{\partial v}\right|_{\varepsilon=\varepsilon_{n}}\right)\right]^{-1 / 2} \times \\
& \times \varphi^{\prime-1 / 2} \sqrt{8 \varphi(\rho)} J_{2 l+1}(\sqrt{8 \varphi(\rho)}) .
\end{aligned}
$$

Using the expression of $\varphi(\rho)$ near the origin given in (A I.27), we obtain our final result :

$$
\begin{aligned}
\lim \rho \rightarrow 0 \frac{R_{n l}(\rho)}{\rho^{l}}=\left[v_{n}^{3}\left(1+\left.\frac{\partial \mu}{\partial v}\right|_{\varepsilon=\varepsilon_{n}}\right)\right]^{-1 / 2} \times \\
\times \frac{2^{l+1} Z^{l+1 / 2}}{(2 l+1) !}
\end{aligned}
$$

$$
\times\left[1-\zeta \frac{l(l+1)(2 l+1)}{6}+O\left(\varepsilon^{2}\right)+O\left(\zeta^{2}\right)\right] .
$$

We are, in fact, only interested in the cases $l=0$ and $l=1$ :

$$
\begin{aligned}
& R_{n 0}(0)=\frac{2 Z^{1 / 2}}{v_{n}^{3 / 2}}\left(1+\left.\frac{\partial \mu}{\partial v}\right|_{\varepsilon=\varepsilon_{n}}\right)^{-1 / 2} \times\left(1+O\left(\varepsilon^{2}\right)\right) \\
&\left.\frac{\mathrm{d} R_{n 1}}{\mathrm{~d} \rho}\right|_{\rho=0}= \frac{2}{3} \frac{Z^{3 / 2}}{v_{n}^{3 / 2}}\left(1+\left.\frac{\partial \mu}{\partial v}\right|_{\varepsilon=\varepsilon_{n}}\right)^{-1 / 2} \times \\
& \times\left[1-\zeta+O\left(\varepsilon^{2}\right)+O\left(\zeta^{2}\right)\right] .
\end{aligned}
$$

\section{APPENDIX II}

In this section, we shall give a relativistic treatment of the parity mixing amplitude in a single particle wave function. The relativistic corrections are expected to be quite large, due to the fact that the Dirac wave functions of an electron moving in the Coulomb field of a point like nucleus are infinite at the origin. This kind of difficulty occurs in the theory of $\beta$-decay and $K$ capture, and is solved by taking into account the finite size of the nucleus. In $\beta$-decay calculations, instead of solving the Dirac equation in the Coulomb field of a finite size nucleus, and averaging the function obtained in this way over the nuclear volume, it has been shown that almost the same results can be obtained by using wave functions relative to a point like nucleus, but evaluated at the surface of the nucleus, i. e. for $r=R$, where $R$ is the nuclear radius. We shall adhere in our treatment to this simple prescription.

The matrix element of the parity violating interaction, which, in the non-relativistic limit, reduces to the potential given in formula (1), is given by :

$$
\begin{aligned}
\left\langle n \mathrm{~s}_{1 / 2}|V|\right. & \left.n^{\prime} \mathrm{p}_{1 / 2}\right\rangle=\frac{i G_{\mathrm{F}} Q_{\mathrm{W}}(Z, A)}{8 \sqrt{2} \pi} \times \\
& \times\left.\left(g_{-1 n} f_{1 n^{\prime}}-g_{1 n^{\prime}} f_{-1 n}\right)\right|_{r=R},
\end{aligned}
$$

where the radial wave functions $f_{x n}$ and $g_{x n}$ are defined in terms of the four component Dirac wave function in a central field by :

$$
n \psi_{j l m}(\mathbf{r})=\left(\begin{array}{ll}
g_{\varkappa n} & \Omega_{j m}^{l} \\
i f_{\varkappa n} & \Omega_{j m}^{l^{\prime}}
\end{array}\right)
$$

with

$x=-l-1$ if $j=l+\frac{1}{2}, \quad x=+l$ if $j=l-\frac{1}{2}$, and $\quad l^{\prime}=2 j-l$.

The radial wave functions $f_{x}$ and $g_{x}$ obey the system of linear differential equations :

$$
\begin{aligned}
& \frac{\mathrm{d} f_{\varkappa}}{\mathrm{d} r}=\frac{\varkappa-1}{r} f_{\varkappa}-\left(W-m_{\mathrm{e}}-V\right) g_{\varkappa}, \\
& \frac{\mathrm{d} g_{\varkappa}}{\mathrm{d} r}=-\frac{\varkappa+1}{r} g_{\varkappa}+\left(W+m_{\mathrm{e}}-V\right) f_{\varkappa} .
\end{aligned}
$$

In this Appendix, it is convenient to use the unit system $\hbar=c=1$. $W$ is the total energy of the electron :

$$
W \simeq m_{\mathrm{e}}+\varepsilon_{n} \frac{m_{\mathrm{e}} \alpha^{2}}{2}
$$

Let $G_{\varkappa}=r g_{\varkappa}$ and $F_{\varkappa}=r f_{\varkappa}$ and eliminate $G_{\varkappa}$ in order to get a second order differential equation for $F_{\varkappa}$ :

$F_{\varkappa}^{\prime \prime}-\frac{b^{\prime}}{b} F_{\varkappa}^{\prime}+F_{\varkappa}\left(-\frac{\varkappa(\varkappa-1)}{r^{2}}+\frac{b^{\prime}}{b} \frac{\varkappa}{r}+a b\right)=0$,

where we have defined :

$$
a=W+m_{\mathrm{e}}-V, \quad b=W-m_{\mathrm{e}}-V .
$$

The prime denotes a derivative with respect to $r$.

In order to get a differential equation looking like a one-dimensional Schrödinger equation, we perform yet another transformation :

$$
F_{\varkappa}=b^{1 / 2} H_{x} .
$$

The differential equation for $H_{\varkappa}$ reads as follows :

$$
\begin{aligned}
\frac{\mathrm{d}^{2} H_{x}}{\mathrm{~d} r^{2}}+H_{x}\left(\frac{-\varkappa(\varkappa-1)}{r^{2}}+\frac{x}{r} \frac{b^{\prime}}{b}+\right. \\
\left.\quad+\frac{1}{2} \frac{b^{\prime \prime}}{b}-\frac{3}{4} \frac{b^{\prime 2}}{b^{2}}+a b\right)=0 .
\end{aligned}
$$

We shall now apply to this Schrödinger-like equation the method of Appendix I.

The non-relativistic approximation is obtained by making the identifications :

$$
\left.\begin{array}{l}
g_{-1 n}^{N . R} \simeq R_{n 0}, \quad g_{1 n^{\prime}}^{N . R} \simeq R_{n^{\prime} 1} \\
f_{1 n^{\prime}}^{N . R} \approx \frac{1}{2 m_{\mathrm{e}}}\left(R_{n^{\prime} 1}^{\prime}+\frac{2}{r} R_{n^{\prime} 1}\right) \\
f_{-1 n}^{N . R} \approx \frac{1}{2 m_{\mathrm{e}}} R_{n 0}^{\prime}
\end{array}\right\} .
$$

The formula (A II.1) reduces to formula (7) provided terms of order $Z R / a_{0} \sim 10^{-2}$ are neglected. 
Let us introduce the quantity :

$$
K\left(r, \varepsilon_{n}, \varepsilon_{n^{\prime}}, Z\right)=\frac{g_{-1 n} f_{1 n^{\prime}}-g_{1 n^{\prime}} f_{-1 n}}{\left(g_{-1 n} f_{1 n^{\prime}}-g_{1 n^{\prime}} f_{-1 n}\right)^{N \cdot R}} .
$$

The relativistic corrections are important in the region where

$$
\frac{V(r)}{m_{\mathrm{e}}} \sim 1, \text { i. e. for } \quad r \sim \frac{Z \alpha}{m_{\mathrm{e}}}=Z \alpha^{2} a_{0} .
$$

For valence electrons, the major part of the wave function lies in the region $V(r) / m_{\mathrm{e}} \lesssim \alpha$. In particular, in the region $r>r_{\mathrm{c}}$, a non-relativistic approximation is certainly adequate, and since the normalization of the wave function, through the quantum defect method, uses only this domain, the outer part of the wave function is not affected by the relativistic correction. The function $K\left(r, \varepsilon_{n}, \varepsilon_{n^{\prime}}, Z\right)$ will differ significantly from unity only for values of $r$ around $Z \alpha^{2} a_{0}$. For such values of $r$, the energy of the valence electron is completely negligible with respect to the potential energy, $V(r)$, so that the function $K\left(r, \varepsilon_{n}, \varepsilon_{n^{\prime}}, Z\right)$ should have a very weak dependence on $\varepsilon$. It is then legitimate to make the approximation :

$$
K_{r}(r, Z)=K\left(r, \varepsilon_{n}=\varepsilon_{n^{\prime}}=0, Z\right) \approx K\left(r, \varepsilon_{n}, \varepsilon_{n^{\prime}}, Z\right) .
$$

Let us first write down a simplified form for the differential equation (A II.6), valid in the region $0 \leqslant r \leqslant 1 / m_{\mathrm{e}} \alpha Z$. The potential $V(r)$ can be approximated by $V(r) \simeq-Z \alpha / r+V_{\mathrm{e}}$, where $V_{\mathrm{e}}$ is the potential of the core electrons at the origin, already introduced in Appendix I, and which can be treated as a perturbation with respect to the nuclear potential $Z \alpha / r$ when $r \lesssim 1 / m_{\mathrm{e}} \alpha Z$. We can use the following approximations :

$$
\begin{gathered}
\frac{b^{\prime}}{b} \approx-\frac{1}{r}-\frac{V_{\mathrm{e}}}{Z \alpha}, \quad \frac{b^{\prime \prime}}{b} \approx \frac{2}{r^{2}}+\frac{2 V_{\mathrm{e}}}{Z \alpha r}, \\
a b \approx \frac{2 m_{\mathrm{e}} Z \alpha}{r}+\frac{Z^{2} \alpha^{2}}{r^{2}}-2 V_{\mathrm{e}}\left(\frac{Z \alpha}{r}+m_{\mathrm{e}}\right) .
\end{gathered}
$$

In the region $0 \leqslant r \lessgtr 1 / Z m_{\mathrm{e}} \alpha$, the differential equation for $H_{x}$ corresponding to $\varepsilon=0$ takes the following simplified form :

$$
\begin{aligned}
H_{\varkappa}^{\prime \prime}+ & H_{\varkappa}\left\{-\frac{1}{r^{2}}\left(\varkappa^{2}-(Z \alpha)^{2}-\frac{1}{4}\right)-2 m_{\mathrm{e}} V_{\mathrm{e}}+\right. \\
+ & \left.\frac{1}{r}\left[2 m_{\mathrm{e}} Z \alpha-2 V_{\mathrm{e}} Z \alpha-\left(\varkappa+\frac{1}{2}\right) \frac{V_{\mathrm{e}}}{Z \alpha}\right]\right\} .
\end{aligned}
$$

Returning now to atomic units by setting $r=\frac{1}{m_{\mathrm{e}} \alpha} \rho$, we get the equation :

$$
\frac{\mathrm{d}^{2} H_{\chi}}{\mathrm{d} \rho^{2}}+H_{\varkappa}\left(\frac{-\lambda(\lambda+1)}{\rho^{2}}+\frac{2 \bar{Z}}{\rho}-2 \zeta Z^{2}\right)=0 .
$$

where $\lambda, \bar{Z}, \zeta$ are given by :

$$
\left.\begin{array}{l}
\lambda=\gamma-\frac{1}{2}=\sqrt{\varkappa^{2}-(Z \alpha)^{2}}-\frac{1}{2} \\
\bar{Z}=Z\left[1-Z^{2} \alpha^{2} \zeta-\frac{1}{2}\left(x+\frac{1}{2}\right) \zeta\right] \\
\zeta=\frac{V_{\mathrm{e}}}{m_{\mathrm{e}} Z^{2} \alpha^{2}}
\end{array}\right\} .
$$

The differential equation looks like a Schrödinger equation for a Coulomb field, but with a non-integral angular momentum. If one eliminates $F_{\chi}$ instead of $G_{\varkappa}$, it is not possible to arrive at such a simple form in the same range of values of $\rho$. In the outer region $\rho>\rho_{\mathrm{c}}$, where relativistic effects are unimportant, the differential equation for $H_{\varkappa}$ reads :

$\frac{\mathrm{d}^{2} H_{\chi}}{\mathrm{d} \rho^{2}}+H_{\varkappa}\left(-\frac{1}{\rho^{2}}\left(\varkappa^{2}-\frac{1}{4}\right)+\frac{2}{\rho}\right)=0$.

It is clear now that we can follow the method developed in Appendix I, with the simplifying feature that we can work in the limit of zero energy. We write $H_{x}(\rho)$ as :

$H_{\varkappa}=N\left(t^{\prime}(\rho)\right)^{-1 / 2} \sqrt{8 t(\rho)} J_{2 \lambda+1}(\sqrt{8 t(\rho))}$,

where the function $t(\rho)$ is given, in the region

$$
0 \leqslant \rho \leqslant \frac{\lambda(\lambda+1)}{2 Z},
$$

by :

$t(\rho)=\bar{Z}\left(1-\zeta \frac{\lambda(\lambda+1)}{3}\right) \rho-\zeta \frac{\bar{Z}^{2} \rho^{2}}{3}+O\left(\zeta^{2}\right)$

and in the region $\rho \gg 1, \rho>\rho_{\mathrm{c}}$, by :

$$
\sqrt{8 t(\rho)}=\sqrt{8 \rho}+\Lambda^{\prime}+O\left(\frac{1}{\rho}\right)
$$

where $\Lambda^{\prime}$ is a constant defined in terms of integrals involving the effective potential appearing in the Schrödinger-like equation (A II.6).

We shall fix the constant $N$ by requiring that for $\rho \gg 1, F_{\varkappa}$ fits the non-relativistic expression computed from the wave function derived in Appendix I :

$$
\begin{gathered}
G_{\varkappa} \approx U_{l} \\
F_{\varkappa}=\frac{1}{a}\left(\frac{\mathrm{d} G_{\varkappa}}{\mathrm{d} r}+\frac{\varkappa}{r} G_{\varkappa}\right) \approx \frac{1}{2 m_{\mathrm{e}}}\left(\frac{\mathrm{d}}{\mathrm{d} r} U_{l}+\frac{\varkappa}{r} U_{l}\right),
\end{gathered}
$$

where

$$
l=x \quad \text { if } \quad x>0, \quad l=-x-1 \text { if } x<0,
$$

and $U_{l}$ is the non-relativistic function given in (A I.34), taken in the limit $\varepsilon=0$. In the limit of large values 
of $\rho$, one finds the following expression for $F_{\varkappa}$ :

$$
\begin{aligned}
F_{\varkappa}= & (-1)^{l} 2 C_{l}(0) \alpha\left(\frac{2}{\pi z}\right)^{1 / 2} \times \\
& \times\left[\sin \left(z+\Lambda^{(0)}+\frac{\pi}{4}\right)+O\left(\frac{1}{z}\right)\right],
\end{aligned}
$$

with $z=\sqrt{8 \rho}$.

This expression has to be compared with the one obtained from (A II.12) in the limit of large values of $\rho$ :

$$
\begin{aligned}
F_{\varkappa}=b^{1 / 2} H_{\varkappa}= & N\left(8 m_{\mathrm{e}} \alpha^{2}\right)^{1 / 2}\left(\frac{2}{\pi z}\right)^{1 / 2} \times \\
& \times \cos \left(z+\Lambda^{\prime}-(2 \lambda+1) \frac{\pi}{2}-\frac{\pi}{4}\right) .
\end{aligned}
$$

Remembering that

$$
2 \lambda+1=2 \gamma=2 \sqrt{\varkappa^{2}-(Z \alpha)^{2}},
$$

we can rewrite $F_{\varkappa}$ as :

$$
\begin{aligned}
F_{\varkappa} & =N\left(8 m_{\mathrm{e}} \alpha^{2}\right)^{1 / 2}\left(\frac{2}{\pi z}\right)^{1 / 2}(-1)^{|\varkappa|} \times \\
& \times \sin \left[z+\Lambda^{\prime}-\pi\left(\sqrt{\varkappa^{2}-(Z \alpha)^{2}}-|\varkappa|\right)+\frac{\pi}{4}\right] .
\end{aligned}
$$

The two expressions coincide if one makes the identification :

$$
\begin{aligned}
\Lambda^{(0)} & =\Lambda^{\prime}-\pi\left(\sqrt{\varkappa^{2}-(Z \alpha)^{2}}-|\varkappa|\right)+k \pi \\
2(-1)^{l} C_{l}(0) & \doteq N\left(8 m_{\mathrm{e}}\right)^{1 / 2}(-1)^{|\varkappa|}(-1)^{k} .
\end{aligned}
$$

If one considers the special case of atomic hydrogen $\left(\Lambda^{(0)}=0, Z=1\right)$, the first equation is satisfied to order $\alpha^{2}$ only if $k=0$. We have finally :

$$
N=\left(2 m_{\mathrm{e}}\right)^{-1 / 2} C_{l}(0)(-1)^{l+|x|} \text {. }
$$

We are now in a position to compute $G_{\varkappa}$ and $F_{\varkappa}$ for $\rho=R / a_{0} \ll 1 / Z$. The function $F_{\chi}$ is simply given by $F_{x}=b^{1 / 2} H_{x}$. When $\rho=R / a_{0} \ll 1 / Z$, it is legitimate to use for $b$ the simplified form :

$$
b \approx \frac{Z \alpha}{r}=\frac{m_{\mathrm{e}} Z \alpha^{2}}{\rho} .
$$

One obtains the following expression :

$$
\begin{aligned}
& \begin{aligned}
F_{x}(R)= & 2 \alpha(-1)^{l+|x|} C_{l}(0) Z^{1 / 2} \times \\
& \times J_{2 \gamma}\left(\sqrt{Z^{*} z}\right)\left(1+O\left(\zeta \frac{Z R}{a_{0}}\right)\right),
\end{aligned} \\
& \text { where }
\end{aligned}
$$

$$
\begin{aligned}
\gamma & =\sqrt{\varkappa^{2}-(Z \alpha)^{2}}=\lambda+\frac{1}{2}, \\
Z^{*} & \approx \bar{Z}\left[1-\frac{\zeta}{3}\left(\gamma^{2}-\frac{1}{4}\right)\right] .
\end{aligned}
$$

The radial wave function $G_{\varkappa}=r g_{\varkappa}$ associated with the large component is readily obtained from eq. (A II.3) :

$$
G_{\varkappa}=\frac{b^{-1}}{r}\left(\varkappa F_{\varkappa}-r \frac{\mathrm{d}}{\mathrm{d} r} F_{\varkappa}\right)
$$

with the approximated form of $b \approx Z \alpha / r$ and the relation $r \frac{\mathrm{d}}{\mathrm{d} r}=\frac{1}{2} z \frac{\mathrm{d}}{\mathrm{d} z}$, one obtains :

$$
G_{\varkappa}=\frac{1}{Z \alpha}\left(\varkappa F_{\varkappa}-\frac{1}{2} z \frac{\mathrm{d}}{\mathrm{d} z} F_{\varkappa}\right) \text {. }
$$

Making use of a well-known identity involving the derivative of a Bessel function, one arrives finally at the following expression for $G_{\chi}(R)$ :

$$
\begin{gathered}
G_{\varkappa}(R)=2 Z^{-1 / 2}(-1)^{l+|\varkappa|} C_{l}(0) \times \\
\times\left[(\varkappa-\gamma) J_{2 \gamma}\left(z \sqrt{Z^{*}}\right)-\frac{1}{2} z \sqrt{Z} * J_{2 \gamma+1}\left(z \sqrt{Z^{*}}\right)\right] .
\end{gathered}
$$

Let us now evaluate the quantity $G_{-1} F_{1}-G_{1} F_{-1}$, keeping only the lowest order term in $Z R / a_{0}$. One finds :

$$
\begin{aligned}
& \left(G_{-1} F_{1}-G_{1} F_{-1}\right)_{r=R}= \\
= & 8 \alpha C_{1}(0) C_{0}(0)\left[\left(\frac{2 Z R}{a_{0}}\right)^{\gamma_{1}} \frac{1}{\Gamma\left(2 \gamma_{1}+1\right)}\right]^{2} \times \\
\times & \left\{1-2 \zeta \gamma_{1}\left[\left(\frac{\gamma_{1}^{2}}{3}+\frac{1}{6}\right)+(Z \alpha)^{2}\right]\right\},
\end{aligned}
$$

with $\gamma_{1}=\sqrt{1-(Z \alpha)^{2}}$.

This expression is to be compared with the nonrelativistic result obtained from (A II.15) :

$$
\begin{aligned}
\left(G_{-1} F_{1}\right. & \left.-G_{1} F_{-1}\right)^{N \cdot R}= \\
& =\frac{1}{2 m_{\mathrm{e}}} U_{0}\left(\frac{\mathrm{d}}{\mathrm{d} r} U_{1}+\frac{1}{r} U_{1}\right) \\
& =8 \alpha C_{1}(0) C_{0}(0)\left(\frac{Z R}{a_{0}}\right)^{2}(1-\zeta) .
\end{aligned}
$$

The two expressions coincide if $(Z \alpha)^{2}$ can be neglected compared to unity. We are now in a position to compute the correcting function :

$$
\begin{gathered}
K_{\mathrm{r}}(R, Z)=\left.\frac{g_{-1} f_{1}-g_{1} f_{-1}}{\left(g_{-1} f_{1}-g_{1} f_{-1}\right)^{N . R}}\right|_{r=R} \\
=\left.\frac{G_{-1} F_{1}-G_{1} F_{-1}}{\left(G_{-1} F_{1}-G_{1} F_{-1}\right)^{N . R}}\right|_{r=R} \\
K_{r}=\left[\left(\frac{2 Z R}{a_{0}}\right)^{\sqrt{1-(Z \alpha)^{2}}-1} \frac{\Gamma(3)}{\Gamma\left(2 \sqrt{1-Z^{2} \alpha^{2}}+1\right)}\right]^{2} \times \\
\times\left\{1-\zeta\left[\sqrt{1-(Z \alpha)^{2}}\left(1+\frac{4}{3}(Z \alpha)^{2}\right)-1\right]\right\} .
\end{gathered}
$$

The second term in brackets corresponds to a small negative correction $0.11 \zeta \simeq 0.01$ for $\mathrm{Cs}$, and $0.18 \zeta \simeq 0.017$ for $\mathrm{Pb}$. We have therefore omitted it in the formula reported in the text. 


\section{APPENDIX III}

In this Appendix, we would like to derive the nonrelativistic form of the parity violating electronnucleus and electron-electron potentials starting from a relativistic current-current interaction. We shall also give an estimate of the matrix element of the electron-electron potential for valence electrons and show that it is smaller than the electron-nucleus potential by a factor $Z$ for large values of $Z(Z>50)$. As a model for neutral currents, we shall use the simplest version of the $\mathrm{SU}_{2} \otimes \mathrm{U}_{1}$ gauge model, unifying weak and electromagnetic interactions. In the limit where the mass of the intermediate neutral vector boson is infinite, one uses an effective currentcurrent Hamiltonian density $\mathcal{H}(x)$ given by :

$$
\mathscr{H}(x)=\frac{G_{\mathbf{F}}}{\sqrt{2}} \mathfrak{J}_{\mu}(x) \mathfrak{J}^{\mu}(x) .
$$

The current $J_{\mu}(x)$ is split into a leptonic part $J_{\mu}^{1}(x)$ and an hadronic part $\mathrm{J}_{\mu}^{\mathrm{h}}(x)$ :

$$
\left.\begin{array}{r}
J_{\mu}^{1}(x)=\frac{1}{2}\left[\bar{v}_{\mathrm{e}}(x) \gamma_{\mu}\left(1+\gamma_{5}\right) v_{\mathrm{e}}(x)+\right. \\
+\overline{\mathrm{e}}(x) \gamma_{\mu} \mathrm{e}(x)\left(4 \sin ^{2} \theta_{\mathrm{w}}-1\right) \\
\left.-\overline{\mathrm{e}}(x) \gamma_{\mu} \gamma_{5} \mathrm{e}(x)+\left(\mathrm{e} \rightarrow \mu, v_{\mathrm{e}} \rightarrow v_{\mu}\right)\right] \\
J_{\mu}^{\mathrm{h}}(x)=J_{\mu, 3}(x)+J_{\mu, 3}^{5}(x)-2 \sin ^{2} \theta_{\mathrm{W}} J_{\mu}^{\mathrm{em}}(x)+J_{\mu}^{\prime}(x)
\end{array}\right\} .
$$

The symbols $\mathrm{e}(x), v_{\mathrm{e}}(x)$, etc... stand for the Dirac fields associated with the electron, neutrino, etc...; $J_{\mu}^{\mathrm{em}}(x)$ is the electric current density for the hadrons, $J_{\mu, 3}(x)$ is the usual hadronic isospin current normalized in such a way that $\int J_{0,3}(x) \mathrm{d}^{3} x$ is the isospin operator $I_{3} ; J_{\mu, 3}^{5}(x)$ is the axial isospin current with the usual current algebra normalization. The current $J_{\mu}^{\prime}(x)$ containing two terms associated with the conservation of charm and strangeness quantum numbers plays no role in the processes considered in this paper.

The electron-nucleus parity violating interaction with no dependence on nuclear spin is described by the following effective Hamiltonian :

$$
\begin{aligned}
& H_{\text {p.v. }}^{\text {e.N. }}=-\frac{G_{\mathrm{F}}}{\sqrt{2}}\left(\mathrm{e}^{\dagger}(x) \gamma_{5} \mathrm{e}(x)\right)_{x=0} \times \\
& \times\left(\frac{Z-N}{2}-2 \sin ^{2} \theta_{\mathbf{w}} Z\right) .
\end{aligned}
$$

To obtain this expression, we have made a monopole approximation, since the wavelength associated with the momentum transfer to the nucleus is much larger than the size of the nucleus. In practice, one should take into account the finite size of the nucleus, since the Dirac Coulomb wave functions for a pointlike charge diverge at the origin. This problem is discussed in detail in Appendix II.
The matrix element of the leptonic axial current component $\left.\left\langle a\left|\mathrm{e}^{\dagger}(\mathbf{x}) \gamma_{5} \mathrm{e}(\mathbf{x})\right| b\right\rangle\right|_{x=0}$, can be written in terms of the Dirac wave functions $\psi_{a}(x)$ and $\psi_{b}(x)$ as :

$$
\left.\left\langle a\left|\mathrm{e}^{\dagger}(x) \gamma_{5} \mathrm{e}(x)\right| b\right\rangle\right|_{x=0}=\left.\psi_{a}^{*}(x) \gamma_{5} \psi_{b}(x)\right|_{x=0} .
$$

In the non-relativistic limit, $\psi_{a, b}$ reduces to :

$$
\psi_{a, b}(x)=\left(\begin{array}{c}
\varphi_{a, b}(x) \\
\frac{\boldsymbol{\sigma} \cdot \mathbf{p}}{2 m_{\mathrm{e}}} \varphi_{a, b}(x)
\end{array}\right),
$$

where $\varphi_{a, b}(r)$ are two-component Pauli-Schrödinger wave functions. Remembering that $\gamma_{5}=-\left(\begin{array}{ll}0 & 1 \\ 1 & 0\end{array}\right)$, one finds immediately :

$$
\begin{aligned}
\left.\psi_{a}^{*}(x) \gamma_{5} \psi_{b}(x)\right|_{x=0}= & \\
=- & -\left[\varphi_{a}^{*}\left(\frac{\boldsymbol{\sigma} \cdot \mathbf{p}}{2 m_{\mathrm{e}}} \varphi_{b}\right)+\left(\frac{\boldsymbol{\sigma} \cdot \mathbf{p}}{2 m_{\mathrm{e}}} \varphi_{a}\right)^{*} \varphi_{b}\right] .
\end{aligned}
$$

The effective Hamiltonian (A III.3) can then be replaced by the non-relativistic potential :

$$
V_{\text {p.v. }}^{\text {e.N. }}=\frac{G_{\mathrm{F}}}{4 \sqrt{2} m_{\mathrm{e}}}\left[\boldsymbol{\sigma} \cdot \mathbf{p} \delta^{3}(\mathbf{r})+\delta^{3}(\mathbf{r}) \boldsymbol{\sigma} \cdot \mathbf{p}\right] Q_{\mathrm{w}},
$$

with

$$
Q_{\mathrm{w}}=-\left[\left(4 \sin ^{2} \theta_{\mathrm{w}}-1\right) Z+N\right] .
$$

Our main concern here is the parity violating electron-electron interaction. In the language of second quantization, the parity violating Hamiltonian reads :

$$
\begin{aligned}
H_{\mathrm{p} . v .}^{\text {e.e. }}=\frac{G_{\mathrm{F}}}{\sqrt{2}}\left(\frac{1}{2}\right. & \left.-2 \sin ^{2} \theta_{\mathrm{w}}\right) \times \\
& \times \int\left(\overline{\mathrm{e}}(x) \gamma_{\mu} \mathrm{e}(x)\right)\left(\overline{\mathrm{e}}(x) \gamma^{\mu} \gamma_{5} \mathrm{e}(x)\right) \mathrm{d}^{3} x .
\end{aligned}
$$

Making a non-relativistic approximation, it is easy to derive a non-relativistic electron-electron potential $V_{\mathbf{p} . v .}^{\text {e.e. }}$ :

$$
\begin{aligned}
V_{\text {p.v. }}^{\text {e.e. }} & =\frac{G_{\mathrm{F}}}{2 \sqrt{2} m_{\mathrm{e}}}\left(2 \sin ^{2} \theta_{\mathrm{w}}-\frac{1}{2}\right) \times \\
& \times \delta^{3}\left(\mathbf{r}_{1}-\mathbf{r}_{2}\right)\left(\sigma_{1}-\sigma_{2}+i \sigma_{1} \wedge \sigma_{2}\right)\left(\mathbf{p}_{1}-\mathbf{p}_{2}\right) \\
& \left.+\left(\sigma_{1}-\sigma_{2}-i \sigma_{1} \wedge \sigma_{2}\right) \cdot\left(\mathbf{p}_{1}-\mathbf{p}_{2}\right) \delta^{3}\left(\mathbf{r}_{1}-\mathbf{r}_{2}\right)\right]
\end{aligned}
$$

Since we are mainly interested in atoms with one valence electron, we have to construct an effective one-particle potential describing the parity violating interaction between the valence electron and the electrons of the closed-shells. It is essential to take into account the exchange contribution between 
the valence electron and the core electrons. In particular, the following identity involving the spin exchange operator $P_{\sigma}$ :

$$
i\left(\sigma_{1} \wedge \sigma_{2}\right)=\left(\sigma_{1}-\sigma_{2}\right) P_{\sigma}
$$

is useful.

Calling $n_{\mathrm{e}}(\mathbf{r})$ the density of the closed shell electrons, the one-particle potential takes the simple form :

$$
\begin{aligned}
V_{\text {p.v. }}^{\text {e.e. }}(\mathbf{r}, \boldsymbol{\sigma})= & \frac{G_{\mathbf{F}}}{2 \sqrt{2} m_{\mathrm{e}}}\left(1-4 \sin ^{2} \theta_{\mathrm{W}}\right) \times \\
& \times\left[\boldsymbol{\sigma} \cdot \mathbf{p} n_{\mathrm{e}}(\mathbf{r})+n_{\mathrm{e}}(\mathbf{r}) \boldsymbol{\sigma} \cdot \mathbf{p}\right] .
\end{aligned}
$$

Since we are interested in heavy atoms $(Z \gg 1)$, we shall use the electronic density given by the ThomasFermi model. We shall also take the distribution corresponding to the neutral atom instead of the once-ionized atom, introducing a relative error of the order of $1 / Z$. Expressed in atomic units, the density $n_{\mathrm{e}}(\rho)$ reads :

$$
n_{\mathrm{e}}(\rho)=\frac{1}{3 \pi^{2}}\left[\frac{2 Z}{\rho} \chi\left(\frac{\rho Z^{1 / 2}}{b}\right)\right]^{3 / 2},
$$

where $b$ is a pure number given by :

$$
b=\frac{1}{2}\left(\frac{3 \pi}{4}\right)^{2 / 3}=0.885 \text {. }
$$

The function $\chi(x)$ is tabulated in Landau and Lifshitz [40]. Let us quote some simple properties of $\chi(x)$ :

$$
\lim _{x \rightarrow 0} \chi(x)=1 ; \quad \lim _{x \rightarrow \infty} x^{3} \chi(x)=144 .
$$

We shall use the electronic density given by the Thomas-Fermi model even for small values of $\rho$ (i. e. $\rho \sim 1 / Z$ ), where it is larger than the actual density. In this way, we shall certainly overestimate the effect of the electron-electron parity violating potential.

We shall take as approximate wave functions those obtained in Appendix I :

$$
R_{n l}=K_{n l} \frac{1}{\rho}\left(\frac{\partial \varphi}{\partial \rho}\right)^{-1 / 2} \sqrt{8 \varphi} J_{2 l+1}(\sqrt{8 \varphi})
$$

with

$$
\begin{aligned}
& K_{n 0}=\frac{1}{4} R_{n 0}(0) Z^{-1 / 2}, \\
& K_{n 1}=\frac{3}{4} R_{n 1}(0) Z^{-3 / 2} .
\end{aligned}
$$

For values of $\rho \lesssim 1 / Z$, where the screening of the nuclear charge is of the order of $10 \%$, the function $\varphi$ is given by the same approximate expression for $\mathrm{s}$ and $\mathrm{p}$ waves :

$$
\varphi(\rho) \simeq Z \rho
$$

When $\rho$ is of the order of $Z^{-1 / 3}$, this approximate form is no longer adequate since the screening of the nuclear charge is important. However, for such values of $\rho$, the centrifugal energy $2 / \rho^{2}$ is already smaller than the potential energy by a factor of the order of $2 \times Z^{-2 / 3}$, which is $<0.2$ when $Z>50$. Thus the functions $\varphi$ for $\mathrm{s}$ and $\mathrm{p}$ waves should stay close to each other, even in this region. Furthermore, since most of the electrons of the ion core are at a distance $\lesssim Z^{-1 / 3}$, where $v(\rho) \gg \varepsilon$, we shall take the zero-energy limit in $\varphi$.

Finally, we shall use the lowest order term of our generalized J.W.K.B. approximation. To summarize, the $s$ and $p$ wave functions are those of (A III.6) with the same function $\varphi(r)$ for $\mathrm{s}$ and $\mathrm{p}$, given by the approximate expression :

$$
\xi=\sqrt{8 \varphi} \approx \int_{0}^{\rho} \sqrt{v(\rho)} \mathrm{d} \rho
$$

In terms of the radial wave function $R_{n l}(\rho)$, the matrix element of the electron-electron parity violating potential reads :

$$
\begin{aligned}
\left\langle n \mathrm{~s}_{1 / 2}\left|V_{\mathrm{p.v.}}^{\text {e.e. }}\right| n^{\prime} \mathrm{p}_{1 / 2}\right\rangle & = \\
= & i \frac{G_{\mathrm{F}}}{2 \sqrt{2} m_{\mathrm{e}}}\left(1-4 \sin ^{2} \theta_{\mathrm{W}}\right) \frac{I}{a_{0}^{4}},
\end{aligned}
$$

with

$$
I=\int_{0}^{\infty} n_{\mathrm{e}}(\rho) \rho^{2}\left[R_{n 0}\left(\frac{2}{\rho}+\frac{\partial}{\partial \rho}\right) R_{n^{\prime} 1}-\frac{\partial R_{n 0}}{\partial \rho} R_{n^{\prime} 1}\right] \mathrm{d} \rho .
$$

Since this matrix element will appear to be smaller by a factor of the order of $Z$ than the matrix element of the electron-nucleus parity violating potential, only a crude estimate of $I$ is needed. In order to do this, we shall split the integral $I$ into two parts :

$$
\begin{gathered}
I=I_{1}+I_{2}, \\
I_{1}=\int_{0}^{\lambda / Z} ; \quad I_{2}=\int_{\lambda / \mathrm{Z}}^{\infty},
\end{gathered}
$$

where $\lambda$ is a positive number of the order of unity.

For small values of $\rho$, the term $\frac{\partial R_{n 0}}{\partial \rho} R_{n^{\prime} 1}$ can be neglected. An overestimate of $I_{1}$ is obtained by replacing the Bessel functions by the first terms of their series expansions for small arguments and by taking $\varphi=Z \rho$. In this approximation, one has :

$$
R_{n 0}\left(\frac{2}{\rho}+\frac{\partial}{\partial \rho}\right) R_{n^{\prime} 1} \approx 3\left(R_{n 0} R_{n^{\prime} 1}^{\prime}\right)_{p=0} .
$$

One obtains immediately the following estimate :

$$
\left|I_{1}\right| \lesssim 3\left|R_{n 0}(0) R_{n^{\prime} 1}^{\prime}(0)\right| \int_{0}^{\lambda / Z}\left[n_{\mathrm{e}}(\rho) \rho^{2}\right] \mathrm{d} \rho .
$$


Using the inequality :

$$
n_{\mathrm{e}}(\rho) \lesssim \frac{1}{3 \pi^{2}}\left(\frac{2 Z}{\rho}\right)^{3 / 2}
$$

one arrives at the result :

$$
\left|I_{1}\right| \lesssim \frac{2}{3 \pi^{2}}\left|R_{n 0}(0) R_{n^{\prime} 1}^{\prime}(0)\right|(2 \lambda)^{3 / 2} .
$$

Let us now turn our attention to $I_{2}$. We write the explicit form of the integrand using (A III.6) and (A III.7). After some manipulation, one obtains :

$$
\begin{aligned}
I_{2}= & 3 Z^{-2} R_{n 0}(0) R_{n^{\prime} 1}^{\prime}(0) \int_{\lambda / Z}^{\infty} \mathrm{d} \rho n_{\mathrm{e}}(\rho) \times \\
& \times\left[\frac{\xi}{2 \xi^{\prime} \rho} J_{1}(\xi) J_{3}(\xi)+\frac{\xi}{4} J_{1}(\xi) J_{3}^{\prime}(\xi)-J_{1}^{\prime}(\xi) J_{3}(\xi)\right],
\end{aligned}
$$

(here $\xi^{\prime}$ stands for $\partial \xi / \partial \rho$ ).

We further transform the above expression using the following identity involving derivatives of Bessel functions :-

$$
J_{1}(\xi) J_{3}^{\prime}(\xi)-J_{1}^{\prime}(\xi) J_{3}(\xi)=\frac{4}{\xi}\left(J_{2}^{2}(\xi)-J_{1}(\xi) J_{3}(\xi)\right)
$$

This gives :

$$
\begin{aligned}
I_{2}=3 Z^{-2}\left(R_{n 0}\right. & \left.R_{n^{\prime} 1}^{\prime}\right)_{\rho=0} \int_{\lambda / Z}^{\infty} \mathrm{d} \rho n_{\mathrm{e}}(\rho) \times \\
\times & {\left[J_{2}^{2}(\xi)+J_{1}(\xi) J_{3}(\xi)\left(\frac{\xi}{2 \xi^{\prime} \rho}-1\right)\right] . }
\end{aligned}
$$

Using an integration by parts, and the fact that $\rho v(\rho)$ is a monotonically decreasing function of $\rho$, one derives the inequality :

$$
\frac{\xi}{2 \xi^{\prime} \rho}>1
$$

Remembering that the modulus of Bessel functions have the following upper bound :

$$
\left|J_{n}(\xi)\right| \leqslant \frac{1}{\sqrt{2}} \quad \text { for } \quad n \geqslant 1
$$

one finally obtains an inequality involving $I_{2}$ :

$$
\left|I_{2}\right| \leqslant \frac{3 Z^{-2}}{4}\left|R_{n 0}(0) R_{n^{\prime} 1}^{\prime}(0)\right|\left|\int_{\lambda / Z}^{\infty} \frac{\mathrm{d} \rho}{\rho} n_{\mathrm{e}}(\rho) \frac{\xi}{\xi^{\prime}}\right|
$$

We have finally :

$$
\begin{aligned}
|I| & \leqslant\left|I_{1}\right|+\left|I_{2}\right| \\
& <\frac{1}{\pi^{2}}\left(\frac{4 \sqrt{2}}{3} \lambda^{3 / 2}+2 \sqrt{2} \lambda^{-1 / 2}\right)\left|R_{n 0}(0) R_{n^{\prime} 1}^{\prime}(0)\right| \\
& <\frac{14}{3 \pi^{2}}\left|R_{n 0}(0) R_{n^{\prime} 1}^{\prime}(0)\right|
\end{aligned}
$$

We are now in a position to estimate the ratio $r$ of the matrix elements of electron-electron and electron-nucleus potentials :

$$
\begin{aligned}
r=\left|\frac{\left\langle n \mathrm{~s}\left|V_{\text {e.e. }}^{\text {p.v. }}\right| n^{\prime} \mathrm{p}\right\rangle}{\left\langle n \mathrm{~s}\left|V_{\mathrm{e} . \mathrm{N} .}^{\text {p.v. }}\right| n^{\prime} \mathrm{p}\right\rangle}\right|< & \\
& <\frac{112}{9 \pi} \frac{\left(4 \sin ^{2} \theta_{\mathrm{W}}-1\right)}{\left[\left(4 \sin ^{2} \theta_{\mathrm{W}}-1\right) Z+N\right]} .
\end{aligned}
$$

Taking $\sin ^{2} \theta_{\mathrm{w}}=0.35, Z=55, N=78$, we find :

$$
r<1.6 \times 10^{-2}
$$

\section{References}

[1] Several comprehensive reviews have appeared recently

LEE, B. W., Proceedings of the XVI International Conference on High Energy Physics, ed. J. D. Jackson and A. Roberts (N. A. L. Batavia Ill. 1972), vol. IV, p. 249

Veltman, M., Invited Talk, Symposium on Electron and Photon Interactions at High Energies, Bonn, Aug. (1973) CERN preprint TH. 1742.

WeinBerg, S., II Conference on Elementary Particles, Aix-enProvence, septembre 1973, J. Physique Colly., 34 (1973) C1-45.

[2] Many authors have in fact contributed to the elaboration of the model :

SChWinger, J., Ann. Phys. 2 (1957) 407.

Bludman, S. A., Nuovo Cimento 9 (1958) 433.

Glashow, S. L., Nucl. Phys. 22 (1961) 579.

SalaM, A. and Ward, J., Phys. Lett. 13 (1964) 168. The introduction of spontaneous symmetry as a mass generating mechanism is due to :

Weinberg, S., Phys. Rev. Lett. 19 (1967) 1964.

Salam, A., Nobel symposium, 1968, N. Svartholm, editor.

[3] HASERT, F. H., et al. (Gargamelle collaboration), Phys. Lett. B46 (1973) 138
[4] Benvetuni, A., et al., Phys. Rev. Lett. 32 (1974) 800.

[5] Private communication from the Gargamelle collaboration.

[6] Bouchiat, C. C. and Bouchiat, M. A., Phys. Lett. 48B (1974) 111.

[7] Curtis-Michel, F., Phys. Rev. 138B (1965) 408.

[8] Prentki, J. and Zumino, B., Nucl. Phys. B47 (1972). LeE, B. W., Phys., Rev. D6 (1972) 1188.

[9] Georgi, H. and Glashow, S. L., Phys. Rev. Lett. 28 (1972) 1495.

Piketty, C. A., Nucl. Phys. B 68 (1973) 44

[10] Kunn, H. G., Atomic Spectra, p. 342-346 (Longman) 1969.

[11] Foldy, L. L., Phys. Rev. 111 (1958) 1093.

[12] Fröman, N. and Fröman, P. O., Phys. Rev. 6A (1972) 2064.

[13] Gupta, R., Happer, W., Lam, L. K. and Svanberg, S., Phys. Rev. 8 (1973) 2792.

Liao, K. H., Gupta, R. and Happer, W., Phys. Rev. 8 (1973) 2811

[14] Ham, F. S., Solid State Physics, ed. F. Seitz and D. Turnbull, (Acad. Press) 1 (1955) 127.

[15] Seaton, M. J., Month. Not. R. Astr. Soc. 118 (1958) 504.

[16] Hermann, F. and Skillman, S., Atomic Structure Calculations (Prentice-Hall, New Jersey) 1963. 
[17] Froese Fisher, Charlotte, Atomic Data 4 (1972) 301.

[18] ÜBeRALl, H., Electron Scattering from Complex Nuclei, p. 212, (Academic Press) 1971.

[19] Lobashov, V. M., et al., Phys. Lett. 25B (1967) 104. LiPson, E. D., et al., Phys. Rev. C5 (1972) 932.

[20] Carrico, J. P., Lipworth, E., Sandars, P. G. H., Stein, T. S. and Weisskopf, M. C., Phys. Rev. 174 (1968) 125. See also Gould, H., Phys. Rev. Lett. 24 (1970) 1091.

[21] Moos, H. W. and Woodworth, J. R., Phys. Rev. Lett. 30 (1973) 775.

[22] Bradley III, L. C. and Wall, N. S., Nuovo Cimento 25 (1962) 48.

[23] Poppe, R., Physica 50 (1970) 48.

[24] Garstang, R. H., J. Res. N.B.S. 68A (1963) 61.

[25] Saloman, E. B. and Happer, W., Phys. Rev. 144 (1966) 7.

[26] Bates, D. R. and DamgaArd, A., Phil. Trans. R. Soc. A242 (1949) 101.

[27] Kusch, P. and Taub, H., Phys. Rev. 75 (1949) 1477 ;

White, C. W., Hughes, W. M., Hayne, G. S., Robinson, H. G., Phys. Rev. 7A (1973) 1178.
Tiedeman, J. S. and Robinson, H. G., in Atomic Physics 3, edited by S. J. Smith and D. K. Walters (Plenum Press New York) 1973.

[28] Feinberg, G. and Sucher, J., Phys. Rev. Lett. 26 (1971) 681 ; Drake, G. W. F., Phys. Rev. 3A (1971) 908.

[29] Abragam, A. and van Vleck, J. H., Phys. Rev. 92 (1953) 1448.

[30] Perl, W., Phys. Rev. 91 (1953) 852.

[31] Phillips, M., Phys. Rev. 88 (1952) 202.

[32] Bouchiat, M. A. and Bouchiat, C. C., to be published.

[33] Margerie, J., C. R. Hebd. Séan. Acad. Sci. B 271 (1970) 209.

[34] Beg, M. A. B. and ZeE, A., Phys. Rev. Lett. 30 (1973) 675. FAYET, P., Prepint Orsay, LPTHE 74/9.

[35] Johnson, W. R., Phys. Rev. Lett. 29 (1972) 1123.

[36] Ruderman, M. A., Phys. Rev. 118 (1960) 1632.

[37] Burhop, E. H. S., The Auger Effect (Cambridge University Press) 1952, p. 118.

[38] Kessler, D., et al., Phys. Rev. Lett. 18 (1967) 1179.

[39] FeInBERG, G. and CHEN, M. Y., Rockefeller University, Report number COO-2232-B-43.

[40] Landau, L. D. and Lifshitz, E. M., Mécanique quantique (Editions de Moscou) 1966, p. 289.

[41] Stone, P. M., Phys. Rev. 127 (1962) 1151. 